OPEN ACCESS

Edited by:

Kasturi Mitra,

University of Alabama at Birmingham,

United States

Reviewed by:

Sofia Avnet,

Rizzoli Orthopedic Institute

(IRCCS), Italy

Richard Richardson

Canadian Nuclear Laboratories

(CNL), Canada

*Correspondence:

Pamela L. Wenzel

pamela.l.wenzel@uth.tmc.edu

Specialty section:

This article was submitted to

Stem Cell Research,

a section of the journal

Frontiers in Cell and Developmental

Biology

Received: 06 September 2020

Accepted: 16 November 2020

Published: 07 December 2020

Citation:

Mohammadalipour A, Dumbali SP and Wenzel PL (2020) Mitochondrial

Transfer and Regulators of Mesenchymal Stromal Cell Function and Therapeutic Efficacy. Front. Cell Dev. Biol. 8:603292. doi: 10.3389/fcell.2020.603292

\section{Mitochondrial Transfer and Regulators of Mesenchymal Stromal Cell Function and Therapeutic Efficacy}

\author{
Amina Mohammadalipour ${ }^{1}$, Sandeep P. Dumbali ${ }^{1}$ and Pamela L. Wenzel ${ }^{1,2,3 *}$ \\ ${ }^{1}$ Department of Integrative Biology \& Pharmacology, McGovern Medical School, University of Texas Health Science Center at \\ Houston, Houston, TX, United States, ${ }^{2}$ Center for Stem Cell and Regenerative Medicine, The Brown Foundation Institute of \\ Molecular Medicine, University of Texas Health Science Center at Houston, Houston, TX, United States, ${ }^{3}$ Immunology \\ Program, MD Anderson Cancer Center UTHealth Graduate School of Biomedical Sciences, Houston, TX, United States
}

Mesenchymal stromal cell (MSC) metabolism plays a crucial role in the surrounding microenvironment in both normal physiology and pathological conditions. While MSCs predominantly utilize glycolysis in their native hypoxic niche within the bone marrow, new evidence reveals the importance of upregulation in mitochondrial activity in MSC function and differentiation. Mitochondria and mitochondrial regulators such as sirtuins play key roles in MSC homeostasis and differentiation into mature lineages of the bone and hematopoietic niche, including osteoblasts and adipocytes. The metabolic state of MSCs represents a fine balance between the intrinsic needs of the cellular state and constraints imposed by extrinsic conditions. In the context of injury and inflammation, MSCs respond to reactive oxygen species (ROS) and damage-associated molecular patterns (DAMPs), such as damaged mitochondria and mitochondrial products, by donation of their mitochondria to injured cells. Through intercellular mitochondria trafficking, modulation of ROS, and modification of nutrient utilization, endogenous MSCs and MSC therapies are believed to exert protective effects by regulation of cellular metabolism in injured tissues. Similarly, these same mechanisms can be hijacked in malignancy whereby transfer of mitochondria and/or mitochondrial DNA (mtDNA) to cancer cells increases mitochondrial content and enhances oxidative phosphorylation (OXPHOS) to favor proliferation and invasion. The role of MSCs in tumor initiation, growth, and resistance to treatment is debated, but their ability to modify cancer cell metabolism and the metabolic environment suggests that MSCs are centrally poised to alter malignancy. In this review, we describe emerging evidence for adaptations in MSC bioenergetics that orchestrate developmental fate decisions and contribute to cancer progression. We discuss evidence and potential strategies for therapeutic targeting of MSC mitochondria in regenerative medicine and tissue repair. Lastly, we highlight recent progress in understanding the contribution of 
MSCs to metabolic reprogramming of malignancies and how these alterations can promote immunosuppression and chemoresistance. Better understanding the role of metabolic reprogramming by MSCs in tissue repair and cancer progression promises to broaden treatment options in regenerative medicine and clinical oncology.

Keywords: cancer metabolism, hematological malignancy, mesenchymal stromal cells (MSCs), MSC differentiation, metabolic reprogramming, mitochondrial biogenesis, mitochondrial dynamics, mitochondrial transfer

\section{INTRODUCTION}

MSCs are multipotent non-hematopoietic cell precursors found in the bone marrow. Albeit highly heterogeneous, similar multipotent stromal cells are ubiquitous in many other tissues throughout the body, such as connective tissue and tumor stroma, and are also referred to as MSCs in the literature (Ullah et al., 2015). MSCs are classical mesodermal derivatives that give rise to chondrocytes, adipocytes, and osteoblasts but are also purported to activate genes required for specialized cells of the ectoderm and endoderm. MSCs have attracted great clinical interest for their ability to differentiate into various cell types, secrete trophic and immunomodulatory factors, and sense and respond to signals generated by inflammation, tissue injury, or tumorigenesis, such as damage associated molecular patterns (DAMPs) (Garg et al., 2010; Krysko et al., 2011). Substantial evidence now suggests that mitochondria play crucial roles as signaling molecules to MSCs and that mitochondrial biogenesis and quality control is vital for MSC function, self-renewal, and differentiation.

Mitochondria are biological powerhouses that provide cellular fuel through conversion of nutrients to energy. In order to meet the demands of cellular activity, mitochondria are responsible for generating the majority of the cell's energy-adenosine triphosphate (ATP)-through oxidative phosphorylation (OXPHOS). In addition to aerobic energy production, mitochondria also play central roles in reactive oxygen species (ROS) production, calcium homeostasis, cellular signaling, and synthesis and/or assembly of metabolites, including fatty acids, amino acids, iron/sulfur clusters, pyrimidines, heme, and steroid hormones (Seo et al., 2018). Mitochondria modulate a range of cellular functions, including apoptosis, autophagy, cell cycle regulation, differentiation, and aging (Rodriguez et al., 2018). As a result, mitochondria are important for cellular adaptation to physiological and pathological microenvironments. Indeed, dysfunction in mitochondria has been linked to cellular aging and several human diseases. Mutations in mitochondrial DNA (mtDNA) or nuclear genes encoding mitochondrial proteins can cause acquired or inherited mitochondrial disease. Less obvious is the mitochondrial dysfunction that occurs in the absence of mutations in mitochondrial genes, which appears as a result of infection, genotoxic insults, or other environmental challenges and can contribute to degenerative disease. Dysfunctional mitochondria produce unhealthy levels of ROS, such as peroxides, superoxide, hydroxyl radical, singlet oxygen, and alpha-oxygen, that damage lipids, protein, and DNA (Li C. et al., 2019). Mitochondrial dysfunction is also tied to ROS-independent factors, including impaired mitochondrial integrity and defective mitochondrial quality control. In a surprising act of altruism, healthy cells can donate or transfer their mitochondria or mtDNA to cells with aged or damaged mitochondria, thus restoring biological fitness of the recipient cell. In this regard, MSCs can exert powerful regenerative effects on aging or injured cells via transfer of mitochondrial cargo.

Despite the therapeutic role of MSCs in tissue homeostasis and repair, MSCs and other stroma can be hijacked for recruitment to tumors to contribute to cancer progression. MSCs can alter metabolism of cancer cells through modification of metabolites and/or nutrients in the microenvironment and by transfer of mitochondria or mtDNA to cancer cells. Mitochondria have diverse effects in tumorigenesis and cancer progression. While some, but not all, cancer cells are heavily dependent upon aerobic glycolysis (Warburg effect), many classical hallmarks of cancer include altered mitochondrial function that extends beyond energy production (Vyas et al., 2016). From cancer initiation to metastasis, mitochondria influence oncometabolite generation, metabolic reprogramming, oxidative signaling, redox homeostasis, cell survival, and metastatic behavior. The ability of MSCs to shelter malignant cells is clinically significant, since MSCs have been shown to reinforce the pro-glycolytic phenotype of leukemia cells and minimize ROS-induced damage to cancer cells (Samudio et al., 2008; Ohkouchi et al., 2012). Furthermore, MSCs have been found to donate mitochondria to solid tumor cells, including breast and lung cancer (Spees et al., 2006; Pasquier et al., 2013). Mitochondrial transfer is also welldocumented in hematological malignancies, such as acute myeloid leukemia (AML), acute lymphocytic leukemia (ALL), and multiple myeloma (MM) (Moschoi et al., 2016; Griessinger et al., 2017; Marlein et al., 2017). In the context of cancer treatment, several studies have revealed that transfer of mitochondria from MSCs can protect mutant hematopoietic cells during chemotherapy.

In this review, we summarize recent progress in the study of mitochondria in MSC differentiation, mitochondrial trafficking mechanisms, and therapeutic applications. We specifically address the mechanisms that mediate mitochondrial transfer and biological consequences of mitochondrial transfer between MSCs and the tumor microenvironment in solid tumors and hematological malignancies. 


\section{MSC DIFFERENTIATION}

The metabolic properties of cells in the bone marrow tightly correlate with their capacity to self-renew, proliferate, endure stress, perform specialized functions, and differentiate. Hematopoietic stem cells (HSCs) and MSCs both reside in the bone marrow, along with their corresponding progenitors at various states of differentiation, mature immune cells, adipocytes, osteoblasts, osteoclasts, stromal cells, endothelial cells, pericytes, and nerve cells. Communication among these cells in various regions of the marrow is crucial in maintenance of hematopoietic stem and progenitor cell homeostasis (Uccelli et al., 2008). The inner lining of the bone surface that interfaces with the marrow, or the endosteum, houses MSCs and other osteoprogenitors. There, MSCs contribute to osteogenesis by interacting with osteoblasts and local hematopoietic cells (Boroumand and Klip, 2020). The bone marrow vasculature also provides distinct microenvironments in the form of sinusoidal and arterial niches, likely characterized by different levels of oxygen tension. Both vessels are lined by endothelial cells but the sinusoid is surrounded by Leptin receptor-expressing stromal cells and arterioles are ensheathed by NG2-expressing periarteriolar cells, sympathetic nerves, and Schwann cells (Asada et al., 2017). These niches modulate HSC quiescence, selfrenewal, and differentiation by direct interaction and release of hematopoietic growth factors and cytokines. Pathological conditions can alter the bone marrow microenvironment, leading to dysregulated differentiation and behavior of MSCs and their progeny. In addition, a substantial body of literature indicates that MSC self-renewal and differentiation not only depend on the cellular composition of the niche, but that they also rely on environmental stimuli from hormones, nutrients, and oxygen. MSCs in the bone marrow experience gradients of oxygen abundance and low oxygen tension, or hypoxia. Undifferentiated MSCs that reside in hypoxic regions of the marrow engage in anaerobic glycolysis, which is essential for survival in hypoxic environments (Mohyeldin et al., 2010). Upon differentiation of MSCs, cells must undergo a metabolic switch away from glycolysis toward oxygen- and mitochondriadependent OXPHOS. Thus, metabolic reprogramming through mitochondrial mediators plays a central role in determining cell fate of MSCs as well as other stem cells. Mitochondrial abundance, morphology, age, activity, fuel usage, and metabolite production can all influence stem cell renewal and fate decisions (Zhang et al., 2018; Bahat and Gross, 2019). Below, we introduce the metabolic reprogramming that accompanies lineage commitment of MSCs and describe mitochondrial features and regulation that participate in this adaptation.

\section{Hypoxia and Metabolism}

Self-renewal of MSCs is thought to be greatest in hypoxic regions of the bone marrow (Mohyeldin et al., 2010). Similarly, long-term plasticity and expansion of ex vivo cultured MSCs is improved under physiological conditions of $2-5 \%$ oxygen (Grayson et al., 2006; Boyette et al., 2014). Adaptation to low oxygen environments is mediated in part by hypoxia-inducible factor- $1 \alpha$ (HIF-1 $\alpha)$, a transcription factor that is stabilized by low oxygen tension (Semenza, 1998). In contrast, high oxygen tension typical of normoxic conditions (20\% oxygen) accelerates proteolytic degradation of HIF- $1 \alpha$, thereby reducing total HIF- $1 \alpha$ levels in the cell. HIF- $1 \alpha$ has been shown to play an essential role in maintenance of MSC stemness and inhibition of terminal differentiation under hypoxia (Yun et al., 2002; Lin et al., 2006). Differentiating MSCs typically undergo a dramatic decrease in glycolysis, concurrent with enhanced mitochondrial respiration (Hofmann et al., 2012; Hsu et al., 2016). HIF- $1 \alpha$ constrains this metabolic reprogramming through transactivation of genes required for anaerobic glycolysis while also suppressing genes necessary for mitochondrial respiration (Semenza, 1998; Kondoh et al., 2007). Thus, HIF-1 $\alpha$ stabilization in low oxygen environments aids in preservation of MSC stemness via inhibition of the metabolic shift to OXPHOS.

Evidence in the literature supports a role for oxygen tension in determination of MSC fate and lineage potential. For example, bone marrow-derived MSCs in three-dimensional (3D) pellet cultures showed the ability to undergo enhanced chondrogenic differentiation in hypoxic conditions, as evidenced by upregulation of cartilage matrix genes and chondrogenesisassociated genes such as the transcription factor SOX6 (Khan et al., 2010). Moreover, fate selection is greatly influenced by changes in oxygen tension. Following normoxic expansion of MSCs, hypoxia amplifies osteogenesis-associated genes, elevates mineral deposition, and enhances chondrogenesis in $3 \mathrm{D}$ pellet cultures, while normoxia inhibits adipogenesis (Boyette et al., 2014). Indeed, severe hypoxia elevates osteoblast lineagespecific transcripts, such as ALPL, the gene that encodes the alkaline phosphatase enzyme important for bone mineralization (Ejtehadifar et al., 2015). Conversely, differentiating MSCs in normoxic conditions express increased levels of adipogenic transcripts (Boyette et al., 2014). In further support of a role for hypoxia in MSC lineage commitment, HIF-1 $\alpha$ knockdown suppresses hypoxia-induced osteogenesis (Wagegg et al., 2012). It is important to note, however, that hypoxia alone is not sufficient to induce expression of all osteoblast-specific transcripts, such as RUNX2, highlighting the importance of other soluble instructive cues in lineage maturation. HIF- $1 \alpha$ has also been shown to be essential for chondrocyte differentiation and survival in physiological hypoxic environments and controls a complex homeostatic response during cartilage and bone development (Araldi and Schipani, 2010).

\section{Mitochondrial Biogenesis}

Bioenergetic demand and capacity evolve as cellular functions change. A striking adaptation in differentiated progeny is the increase in mitochondrial capacity and efficiency. During osteogenesis and adipogenesis, mitochondrial membrane potential, respiratory enzyme complexes, oxygen consumption, and intracellular ATP content are all elevated (Chen et al., 2008; Tahara et al., 2009; Pietilä et al., 2012). Osteogenic induction appears to also induce mitochondrial biogenesis and increase mtDNA copy number (Chen et al., 2008; Pietilä et al., 2012). Interestingly, mtDNA copy number steadily increases over the course of osteogenic maturation and enhances mitochondrial biogenesis (Chen et al., 2008). Consistent with a decreased 
dependence upon glycolysis, these cells also exhibit reduced lactate production. Notably, mitochondrial mass does not appear to be increased during chondrogenesis, highlighting specificity in the bioenergetic requirements of different lineages (Forni et al., 2016). Adaptations in mitochondrial abundance and dynamics in MSCs during lineage commitment are depicted in Figure 1.

Consistent with an increased reliance on OXPHOS, regulators of mitochondrial biogenesis are altered during MSC differentiation. Peroxisome proliferator-activated receptor gamma coactivator 1 -alpha $(\mathrm{PGC}-1 \alpha)$ is a master regulator of mitochondrial biogenesis. PGC- $1 \alpha$ and mtDNA content are both increased in human bone marrow MSCs undergoing adipogenic differentiation (Fernandez-Marcos and Auwerx, 2011; Huang et al., 2011; Hofmann et al., 2012; Zhang et al., 2013). PGC-1 $\alpha$ knockdown inhibits adipogenesis; whereas, PGC- $1 \alpha$ overexpression significantly increases expression of genes involved in mitochondrial functions, lipid metabolism, and brown adipose tissue commitment (Huang et al., 2011). PGC- $1 \alpha$ activates nuclear respiratory factors (NRF1 and NRF2) which, in turn, modulate mitochondrial transcription factor A (TFAM) (Ventura-Clapier et al., 2008). Together, TFAM and DNA polymerase $\gamma$ are essential for replication of the mitochondrial genome (Ekstrand et al., 2004). Upon osteogenic induction of MSCs, increased PGC-1 $\alpha$ is accompanied by increases in TFAM and DNA polymerase $\gamma$ (Chen et al., 2008). Upregulation of TFAM has also been reported during adipogenic and chondrogenic differentiation of MSCs (Chen et al., 2008; Zhang et al., 2013). Interestingly, knockdown of TFAM suppresses mitochondrial activity and blocks adipogenic differentiation of MSCs (Zhang et al., 2013). In this study, TFAM knockdown was accompanied by a significant decrease in MtND2 (TFAM-dependent mitochondrial gene), while CytC (TFAMindependent gene) remained unchanged, indicating that TFAM is a key activator of mitochondrial transcription and genome duplication in MSC lineage commitment (Zhang et al., 2013). Two pathways believed to lie upstream of PGC- $1 \alpha$ regulation in MSCs include Wnt-MAPK signaling and the AMP-activated protein kinase (AMPK) energy and nutrient sensor pathway. In a study of Wnt-induced osteoblastic differentiation of embryonic MSCs, An et al. (2010) revealed that mitochondrial biogenesis plays an important role in the osteoblastic differentiation of mesenchymal progenitor cells. Wnt $/ \beta$-catenin signaling potently induced PGC-1 $\alpha$, TFAM, and mitochondrial biogenesis through activation of the Erk and p38 MAPK pathways (An et al., 2010). In a second study of genetically modified MSCs not undergoing differentiation, ectopic expression of AMPK triggered upregulation of PGC- $1 \alpha$ and TFAM (De Meester et al., 2014). From these data, we conclude that mitochondrial metabolism of differentiating and self-renewing MSCs is greatly influenced by regulation of PGC- $1 \alpha$, mtDNA copy number, and mitobiogenesis.

\section{Mitochondrial Dynamics}

Mitochondria also change shape to meet cellular demands. Cycles of mitochondrial fusion and fission, motility, and cristae remodeling, collectively referred to as mitochondrial dynamics, regulate and can be affected by mitochondrial activity (Cogliati et al., 2016; Mishra and Chan, 2016). Further, deep invaginations in the inner mitochondrial membrane (IMM) known as cristae exhibit profound diversity in structure and composition (Perkins et al., 1997; Zick et al., 2009). Mitochondrial machinery, including electron transport chain complexes, surround and are embedded within the membranes of cristae and thus cristae topology influences respiratory efficiency by regulating assembly of respiratory complexes and supercomplexes, nucleoid maintenance, iron-sulfur biogenesis, and protein synthesis and translocation (Gilkerson et al., 2003; Vogel et al., 2006; Cogliati et al., 2013; Kopek et al., 2013).

Mitochondrial remodeling accompanies fate commitment of both embryonic and adult stem cells. In undifferentiated MSCs, mitochondria localize near the nucleus (perinuclear) and adopt a fragmented spherical shape (Hofmann et al., 2012; Quinn et al., 2013). As MSCs differentiate and mature, a branched network of elongated mitochondria forms uniformly throughout the cytoplasm during osteogenesis and adipogenesis (Hofmann et al., 2012; Quinn et al., 2013). Conversely, chondrogenesis is associated with fragmented spherical mitochondria (Forni et al., 2016; Seo et al., 2018). In addition, a feature unique to terminally differentiated adipocytes is mitochondrial enrichment around lipid droplets (Hofmann et al., 2012), a phenomenon that positions mitochondria closer to respiratory substrates in the lipids and is thought to be particularly relevant in times of nutrient depletion (Rambold et al., 2015). These changes in morphology and distribution occur in conjunction with a shift in metabolism from glycolytic to oxidative, resulting in higher intracellular levels of ATP and ROS (Figure 1). Careful characterization of ultrastructure remains incompletely described in MSCs, but, based upon studies of other stem cells, it is probable that cristae shape and density are also remodeled to support increased capacity for ATP synthesis via modulation of supercomplex assembly and stability (Cogliati et al., 2013).

Morphology and ultrastructure of mitochondria are controlled by numerous mitochondrial-shaping proteins, including GTP-dependent dynamin-like proteins involved in fusion and fission cycles, as well as structural proteins (Pernas and Scorrano, 2016). Mitochondrial division, or fission, requires the actin cytoskeleton and endoplasmic reticulum (ER), along with cytoplasmic partners and adaptor proteins. Mounting evidence supports a model for fission in which the actin cytoskeleton pre-constricts mitochondria at ER contact sites (Hatch et al., 2014). ER-bound inverted formin 2 (INF2) is essential for mediating actin polymerization at these sites, thus triggering a transfer of calcium ions from the ER across the outer mitochondrial membrane (OMM). Elevated matrix calcium activates IMM constriction (Chakrabarti et al., 2018). Actin polymerization also stimulates recruitment of another central player in fission, dynamin-1-like protein (Drp1). Subsequent to IMM division, Drp1 forms oligomer rings around the mitochondria to promote OMM division (Ji et al., 2015). These sites are occupied by a host of other pro-fission and adaptor proteins, including mitochondrial fission protein 1 (Fis1), mitochondrial fission factor (Mff), and mitochondrial dynamics proteins of $49 \mathrm{kDa}$ and $51 \mathrm{kDa}$ (MiD49 and MiD51) which also contribute to loading of Drp1 on fission sites (Losón et al., 


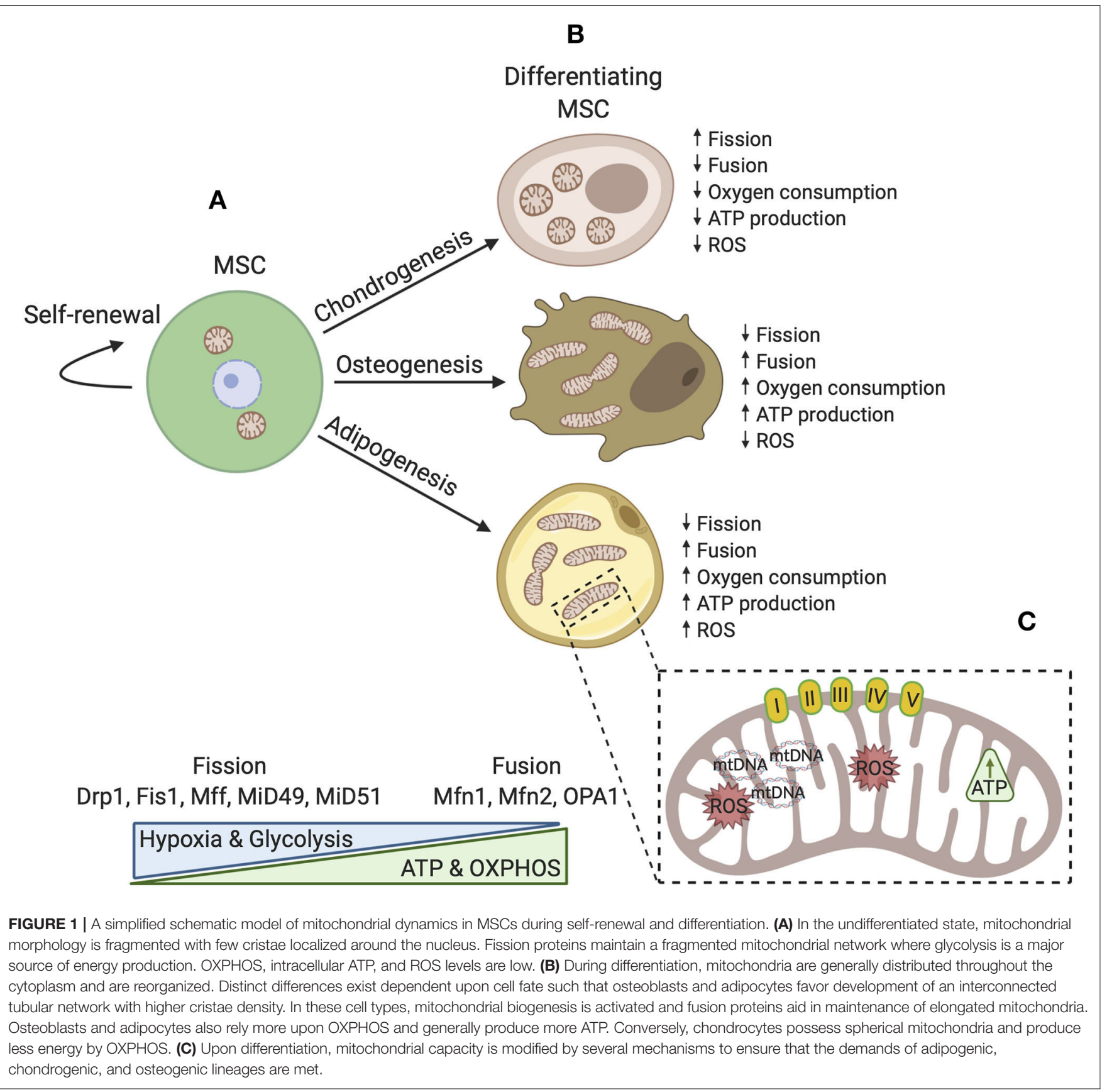

2013) (Figure 1A). Murine dermis-derived MSCs undergoing chondrogenesis express more Drp1, Fis1, and Fis2, consistent with their fragmented mitochondrial morphology (Forni et al., 2016). Using an assay based on dilution of a mitochondrially targeted photoactivatable green fluorescent protein, Forni et al. (2016) observed that chondrogenic MSCs undergo fewer fusion events than both undifferentiated MSCs and MSCs differentiated under osteo- and adipogenic conditions. Further support for the importance of mitochondrial remodeling comes from overexpression of a dominant negative Drp1, which resulted in a reduced ability to induce mitochondrial fragmentation and decreased expression of Col2a and Sox9, two genes important for cartilage formation (Hirao et al., 2006; Eslaminejad et al., 2013; Forni et al., 2016). This suggests that reorganization of the mitochondrial network toward a fragmented spherical phenotype plays an active role in commitment of MSCs to the chondrocyte lineage.

Similarly, specification of osteocytes and adipocytes is influenced by factors that drive fusion of mitochondria. The fusion of mitochondria requires carefully orchestrated tethering of the OMM of each mitochondrion, followed by fusion of the IMMs. Several GTPase proteins participate in 
tethering and mixing of mitochondrial contents, including optic atrophy protein 1 (OPA1), mitofusin-1 (Mfn1), and mitofusin-2 (Mfn2) (Cipolat et al., 2004) (Figure 1B). Along with development of an interconnected tubular mitochondria network, $M f n 1$ and $M f n 2$ transcripts are upregulated in the early steps of osteogenesis and adipogenesis (Forni et al., 2016; Seo et al., 2018). Knockdown of $M f n 2$ results in reduced mitochondrial fusion and oxygen consumption, which causes impaired differentiation (Forni et al., 2016). Specifically, $M f n 2$ knockdown blocked deposition of calcified matrix and expression of genes activated upon development of preosteoblasts into mature osteoblasts, Ost, Runx2, and Dlx5. Likewise, adipocytic cultures produced far less lipid droplets and had reduced expression of genes encoding adipocyte transcription factors, peroxisome proliferation-activated receptor $\gamma(\operatorname{PPAR} \gamma), \mathrm{Rxr}$, and Creb. There is also evidence to suggest that cristae morphology is critical for osteogenic fate and maintenance. Knockdown of the gene that encodes a master regulator of cristae dynamics, Mitofilin (encoded by Immt), which is a core component of the mitochondrial contact site and cristae-organizing system (MICOS) complex, produces giant mitochondria with high IMM to OMM ratios, low mitochondrial membrane potential, decreased ATP generation, and elevated ROS. The insufficiency in Mitofilin disrupts osteogenesis and causes weak alkaline phosphatase activity, reduces mineralization capability, and downregulates expression of osteogenic marker genes, Runx2, Osx, and Ocn (Lv et al., 2018). Collectively, these data implicate mitochondrial morphology and cristae architecture in fate specification of MSCs.

\section{Reactive Oxygen Species}

Reactive oxygen species (ROS) are chemical species containing oxygen that readily react with organic substrates, including lipids, proteins, sugars, and nucleic acids. ROS are primarily generated from NADPH oxidase in the cytoplasmic membrane and enzyme complexes of the electron transport chain (Collin, 2019). Other sources include enzymes in the endoplasmic reticulum (xanthine oxidase, lipo- and cyclo-oxygenase, cytochrome P450) and peroxisomes (Collin, 2019).

Aging and oxidative stress have been reported to cause cellular dysfunction by impacting MSC mitochondrial dynamics (Stab et al., 2016). Long-term MSC culture results in changes in mitochondrial morphology, decreased antioxidant capabilities, and increased ROS levels (Geißler et al., 2012). A comparison between young and old MSCs in culture illustrates that aging enhances mitochondrial fusion through downregulation of Drp1 and upregulation of Mfn2 (Li X. et al., 2019). On the other hand, oxidative stress induced by ROS overgeneration results in mitochondrial fission and fragmentation in MSCs. Conversely, a combination of $\mathrm{N}$-acetyl-l-cysteine (NAC) and ascorbic acid 2-phosphate reduces ROS along with inhibition of mitochondrial fission (Li et al., 2015). Similarly, oxidative stress induced by serum deprivation and hypoxia in MSCs prompts mitochondrial fission associated with upregulation of Drp1 and downregulation of Mfn2 expression (Deng et al., 2020).
Several studies have also examined the role of ROS and related signaling pathways in the regulation of MSC differentiation and senescence. Indeed, although mitochondrial activity and ROS generation are elevated during MSC differentiation, it remained unclear whether these metabolic adaptations were essential for differentiation or simply byproducts of the differentiation process. Tormos et al. (2011) tested the importance of ROS in the differentiation of adipocytes by targeting antioxidants to the mitochondria of human MSCs. They found that antioxidants inhibited adipocyte differentiation, while addition of exogenous hydrogen peroxide could rescue adipogenesis. Further, authors identified the source of the proadipogenic ROS as mitochondrial complex III. Ablation of complex III function through knockdown of one of its essential components, Rieske iron sulfur protein (RISP), revealed that superoxide generated by this complex is necessary to initiate adipocyte differentiation. Interestingly, treatment of MSCs with antimycin $\mathrm{A}$, which diminishes oxygen consumption but permits superoxide generation, did not interfere with upregulation of adipogenic markers such as PPAR $\gamma$. This pro-adipogenic effect of superoxide could have implications for expansion of adipocytes in the marrow, such as is typical during aging (Almeida and O’Brien, 2013; Hu et al., 2018), after myeloablation (Kiang, 2016), and in AML (Hole et al., 2013; Shafat et al., 2017; Sillar et al., 2019). For example, increased levels of superoxide have been reported in 65\% of primary AML blasts compared to normal bone marrow samples (Hole et al., 2013). Thus, various metabolic conditions that intensify stress conditions in the bone can result in enhanced marrow adiposity. Overall, this study provides compelling evidence that ROS generation is not simply a consequence of differentiation but rather is an effector of adipocyte differentiation. Subsequent studies corroborate these conclusions. For example, Lin et al. (2018) found that lipid droplet formation in the mouse MSC line C3H10T1/2 was improved with hydrogen peroxide treatment, while calcium deposition as an indicator of osteogenesis was dramatically reduced. Consistent with the trend toward enhanced adipogenesis and repressed osteogenesis, authors showed upregulation in early and late adipogenic transcription factors KLF5, C/EBPb, PPARc2 and LEPTIN and downregulation of osteogenic transcription factors RUNX2, c-MAF and COL1A. Conversely, these effects were reversed by the ROS scavenger $\mathrm{N}$-acetyl-1-cysteine (NAC).

\section{Sirtuins in Metabolic Reprogramming}

Metabolic reprogramming of differentiating MSCs is accomplished in part by post-translational modification of proteins required for MSC maintenance and differentiation (Li et al., 2011; Xiao and Chen, 2012; Jeon et al., 2016). Post-translational modification in the mitochondria and proteinprotein interactions that modify diverse mitochondrial functions are impacted by cellular demand, nutrient availability, and redox conditions. The sirtuin family of $\mathrm{NAD}^{+}$-dependent protein deacetylases plays a central role in this sensing and regulates cytoplasmic enzymes, chromatin state, and other proteins that dictate response. Seven mammalian sirtuins regulate transcriptional repression, recombination, cell cycle, 
microtubule organization, and cellular response to DNAdamaging agents (North and Verdin, 2004). SIRT1, SIRT6, and SIRT7 catalyze post-translational modification of proteins in the nucleus. SIRT3, SIRT4, and SIRT5 act chiefly on proteins within the mitochondria. SIRT2 primarily localizes to the cytosol, though it can transiently shuttle to the nucleus in a cell cycle-dependent manner. Here, we summarize the role of these sirtuins in metabolic reprogramming of MSCs.

SIRT1 is perhaps the most well-studied sirtuin. Substantial evidence supports roles for SIRT1 in energy metabolism and a variety of physiologic and pathologic events, cell survival, differentiation, and age-related alterations. In the content of aging, activation of SIRT1 by inhibition of age-induced miR195 reverses the senescent phenotype of old MSCs and restores capacity for cell proliferation (Okada et al., 2016). MSC-specific knockout of Sirt1 in mice results in defects that progress with age, including decline in subcutaneous fat and bone mass, phenotypes which originate from impaired maintenance of osteoblasts and chondrocytes (Simic et al., 2013). Pathology could be rescued by expression of mutant $\beta$-catenin that mimics the deacetylated form of $\beta$-catenin, strongly suggesting that $\beta$-catenin is a Sirt1 target that promotes transcription of genes required for MSC differentiation. SIRT1 is upregulated in osteogenic cultures of bone marrow MSCs and continues to increase progressively with maturation of osteoblasts ( $\mathrm{Li} \mathrm{M}$. et al., 2018). Oxidative stress can inhibit this increase in SIRT1, along with reduction in alkaline phosphatase activity and mineralization of matrix, two indicators of early and late differentiation, respectively. Yet, pharmacological activation of SIRT1 by resveratrol can protect against oxidative stress and restore mineralization and expression of early- and late-stage differentiation markers (Li M. et al., 2018). Collectively, numerous studies demonstrate roles for SIRT1 in MSCs and their progeny.

SIRT2 appears to have some shared functions with SIRT1, as SIRT2 has also been reported to have an inhibitory effect on adipogenesis of MSCs. SIRT2 deacetylates FOXO1, thus blocking PPAR $\gamma$ expression (Jing et al., 2007; Wang and Tong, 2009; Si et al., 2013). Although SIRT2 is the most abundant of all sirtuins in adipocytes and pre-adipocytes, its mRNA is down-regulated at the initial stages of differentiation in 3T3L1 cells (Jing et al., 2007). In fact, silencing of Sirt2 accelerates adipocyte differentiation, evidenced by more rapid accumulation of lipids in Sirt2 knockdown cells (Jing et al., 2007). Nucleocytoplasmic shuttling of SIRT2 regulates its activity and, within the cytosol, SIRT2 is poised to interact with cytosolic and cytoskeletal proteins. For this reason, SIRT2 has been implicated in regulation of cellular morphology through deacetylation of $\alpha$-tubulin (Inoue et al., 2007; Nahhas et al., 2007). Remodeling of the cytoskeleton via $\alpha$-tubulin acetylation is crucial for adipocyte development, thus SIRT2 deacetylase activity must be downregulated to permit the cellular reorganization necessary for adipogenesis (Yang et al., 2013).

Three sirtuins, SIRT3, SIRT4, and SIRT5, reside in the mitochondria and regulate the acetylation landscape of mitochondrial proteins. Recent proteomic analyses have also revealed novel modifications targeted by these sirtuins, including succinylation, glutarylation, and malonylation (Carrico et al.,
2018). SIRT3 is responsible for deacetylation of the majority of proteins in the mitochondria; whereas, data suggests that SIRT4 and SIRT5 modify low-abundance acetylated proteins or those with other lysine modifications (Lombard et al., 2007). SIRT3 is a major regulator of mitochondrial biogenesis and function in MSCs. Expression of SIRT3 increases in osteoblastic MC3T3-E1 cells (Ding et al., 2017) and over the course of MSC adipogenic differentiation (Hsu et al., 2016). Consistent with positive regulation of differentiation, silencing SIRT3 in MSCs impairs expression of adipo-lineage markers and adiponectin secretion (Hsu et al., 2016). These phenotypic defects are accompanied by decrease in mitochondrial biogenesis and downregulation of PGC- $1 \alpha$, antioxidant enzymes, TFAM, and protein subunits of respiratory enzyme complexes (Hsu et al., 2016). SIRT3 deficiency impairs mitochondrial respiration, inducing a metabolic shift toward glycolysis. SIRT5 modifies the same protein and lysine residue targets as SIRT3 and is the only sirtuin known to catalyze desuccinylation and demalonylation of mitochondrial proteins (Du et al., 2011; Park et al., 2013; Rardin et al., 2013). Unlike SIRT3, SIRT5 expression is reduced during adipogenic differentiation of human MSCs and thus it remains unclear whether SIRT3 and SIRT5 act competitively or synergistically to regulate metabolism (Hsu et al., 2016).

Lastly, SIRT6 and SIRT7 catalyze modification of proteins located in the nucleus and play critical roles in modulating metabolic homeostasis, genome stability, and DNA repair. The majority of research in human MSCs has centered on the roles of these sirtuins in aging. For example, SIRT7 expression declines during human MSC aging, and SIRT7 deficiency accelerates senescence via loss of heterochromatin in regions of innate immune regulation (Bi et al., 2020). In addition, SIRT6 (Chen et al., 2017) and SIRT7 (Cioffi et al., 2015) have been shown to positively regulate adipogenesis. More recent reports using ex vivo cultures of MSCs corroborate the importance of SIRT6 in adipocyte maturation from mesenchymal progenitors (Sun et al., 2014; Zhang et al., 2017).

\section{Mitophagy}

Mitochondrial function depends strictly on mitochondrial integrity and quality control. Autophagy of mitochondrial components plays an important role in stem cell maintenance by selective sequestration and degradation of dysfunctional or aged mitochondria. Machinery that participates in clearance of mitochondria is specific to the triggering event, such as differentiation, oxygen imbalance, mitochondrial damage, or, in the context of the germline, removal of paternal mitochondria after fertilization (Pickles et al., 2018). Mitochondrial proteins and other cargo are cleared by the autophagosome. Other quality control mechanisms that contribute to turnover of mitochondria include surveillance by the unfolded protein response, shedding of vesicles, proteolysis, and degradation of mitochondrial proteins by the proteasome (Pickles et al., 2018). Standard culture conditions expose MSCs to excess oxygen (21\%) and result in significant increase in mitochondrial ROS production (Phinney et al., 2015). Phinney et al. (2015) found that this oxidative stress induces MSCs to release large vesicles containing entire mitochondria and mitochondrial proteins and 
structures, such as ATP synthase, OMM, IMM, and cristae. The oxidative stress caused by prolonged exposure to high oxygen induces the Pink1/Parkin-mediated pathway of mitophagy. In addition, a standard marker for autophagosomes, the cytosolic microtubule-associated protein light chain 3 (LC3-I), is found in the mitochondria-containing vesicles, further supporting that mitophagy is stimulated by hyperoxia. Support for a role for mitophagy in differentiation of MSCs is more limited but has been found in the early stages of MSC differentiation, particularly toward the chondrocytic fate (Forni et al., 2016).

Collectively, a body of literature show that differentiation of MSCs is accompanied by and depends upon metabolic reprogramming. Upon fate commitment, several lineages amplify their mitochondrial capacity through replication of mtDNA, increased mitobiogenesis, architectural and morphological reorganization, elevated OXPHOS, and greater ROS generation. Rather than a simple consequence of the differentiation process, these adaptations appear to be necessary for initiation of commitment and lineage maturation. Mitochondrial quality control through autophagy is critical in normal mitochondrial and cellular function. In a broader context, mitochondrial dysfunction is one of the hallmarks of cellular damage and is associated with many human diseases. MSCs hold great potential for use in cell therapies not only because of their multipotency, immunomodulation, and trophic functions but also due to their capacity for mitochondrial transfer to damaged cells. However, the detailed mechanisms and conditions required for efficient mitochondrial transfer between MSCs and recipient cells remain incompletely understood. In the next section, we detail our current understanding of how MSCs transfer mitochondria to regulate metabolism in neighboring cells and their microenvironment.

\section{MITOCHONDRIAL TRANSFER}

Mitochondria can acquire damage as a result of aging, inflammation, injury, genotoxic agents, and oxidative stress. Horizontal transfer of mitochondria or mitochondrial genomes between cells can rescue and rebuild biological function in recipient cells. The first report suggesting that mitochondria could be transported spontaneously between cells described exchange of MitoTracker-positive structures from cardiomyocytes to endothelial cells through transient cytoplasmic bridges or nanotubular structures (Koyanagi et al., 2005). Conclusive evidence for horizontal mitochondrial transfer between cells was published the following year, showing that human bone marrow MSCs could rescue aerobic respiration in cells depleted of mtDNA and mitochondria by ethidium bromide (Spees et al., 2006). In the context of injury and inflammation, MSCs respond to ROS and damage-associated molecular patterns (DAMPs), such as damaged mitochondria and mitochondrial products, by donation of their mitochondria to injured cells (Krysko et al., 2011; Paliwal et al., 2018). Through intercellular mitochondria trafficking, modulation of ROS, and modification of nutrient bioavailability, endogenous MSCs and MSC therapies are believed to exert protective effects by regulation of cellular metabolism in injured tissues. Below, we outline the key players that mediate mitochondrial transfer to cells within damaged tissues from MSCs or stromal derivatives of MSCs. We additionally address therapeutic applications of horizontal mitochondrial transfer by MSCs in regenerative medicine. Other comprehensive reviews are available that address therapeutic applications of mitochondrial transfer for conditions such as mitochondrial-related diseases (Torralba et al., 2016) and the preclinical evidence for the trophic and protective effects of MSC intracellular components (Akyurekli et al., 2015), thus these aspects will not be addressed in detail.

\section{Transfer Mechanisms}

Cells utilize various mechanisms for transfer of their mitochondrial cargo to other cells, including tunneling nanotubes, gap junctions, extracellular vesicles, and cell fusion. These processes are depicted in Figure 2 and detailed below.

\section{Tunneling Nanotubes}

Studies of intercellular mitochondrial trafficking are dominated by the role of tunneling nanotubes (TNTs) or membrane nanotubes. TNTs were originally described by (Rustom et al., 2004) as conduits through which mammalian cells can arrange selective transfer of membrane vesicles and organelles between cells. Literature now documents roles for TNTs in exchange of an array of organelles, such as mitochondria, lysosomes, endosomal vesicles, as well as plasma membrane components, lipid droplets, ions, proteins, miRNAs, and pathogens (Gerdes et al., 2007; Davis and Sowinski, 2008). TNT communication allows unidirectional exchange (Rustom et al., 2004; Bukoreshtliev et al., 2009) or bidirectional transfer (He et al., 2011). TNTs form between connecting cells through protrusions of the membrane and have lifetimes ranging from a few minutes to several hours (Gurke et al., 2008). TNTs are $\sim 50-200 \mathrm{~nm}$ in diameter and can extend toward neighboring cells at long distances of over $100 \mu \mathrm{m}$ (Rustom et al., 2004). TNTs contain cytoskeletal filaments composed of F-actin (thin TNT $<0.7 \mu \mathrm{m}$ in diameter) (Rustom et al., 2004) or both F-actin and microtubules (thick TNT $>0.7 \mu \mathrm{m}$ in diameter) (Önfelt et al., 2006). TNTs containing intermediate filaments and microtubules have also been reported (Resnik et al., 2019). Thin TNTs can transfer portions of plasma membrane between cells, while thick TNTs can carry components of the cytoplasm such as vesicles and organelles, including entire mitochondria. TNT-mediated transfer of mitochondria or mtDNA from MSCs to cells of other organs has been reported in respiratory (Pilling et al., 2006), cardiovascular (Cselenyák et al., 2010), neuronal (Babenko et al., 2015), and immune system (Jackson et al., 2016) diseases and disorders (Table 1).

A growing number of studies show that donor cells, including MSCs, must sense cellular damage to exploit TNTs for mitochondrial transport. In human umbilical cord vein endothelial cells exposed to ischemia-reperfusion, TNT generation is dependent upon the ability of MSCs to detect phosphatidylserine (PS) domains on the plasma membrane of the stressed cells (Liu et al., 2014). PS is chiefly oriented toward the cytoplasm on the plasma membrane but moves to the outer leaflet of the membrane (cell surface) during early stages of 


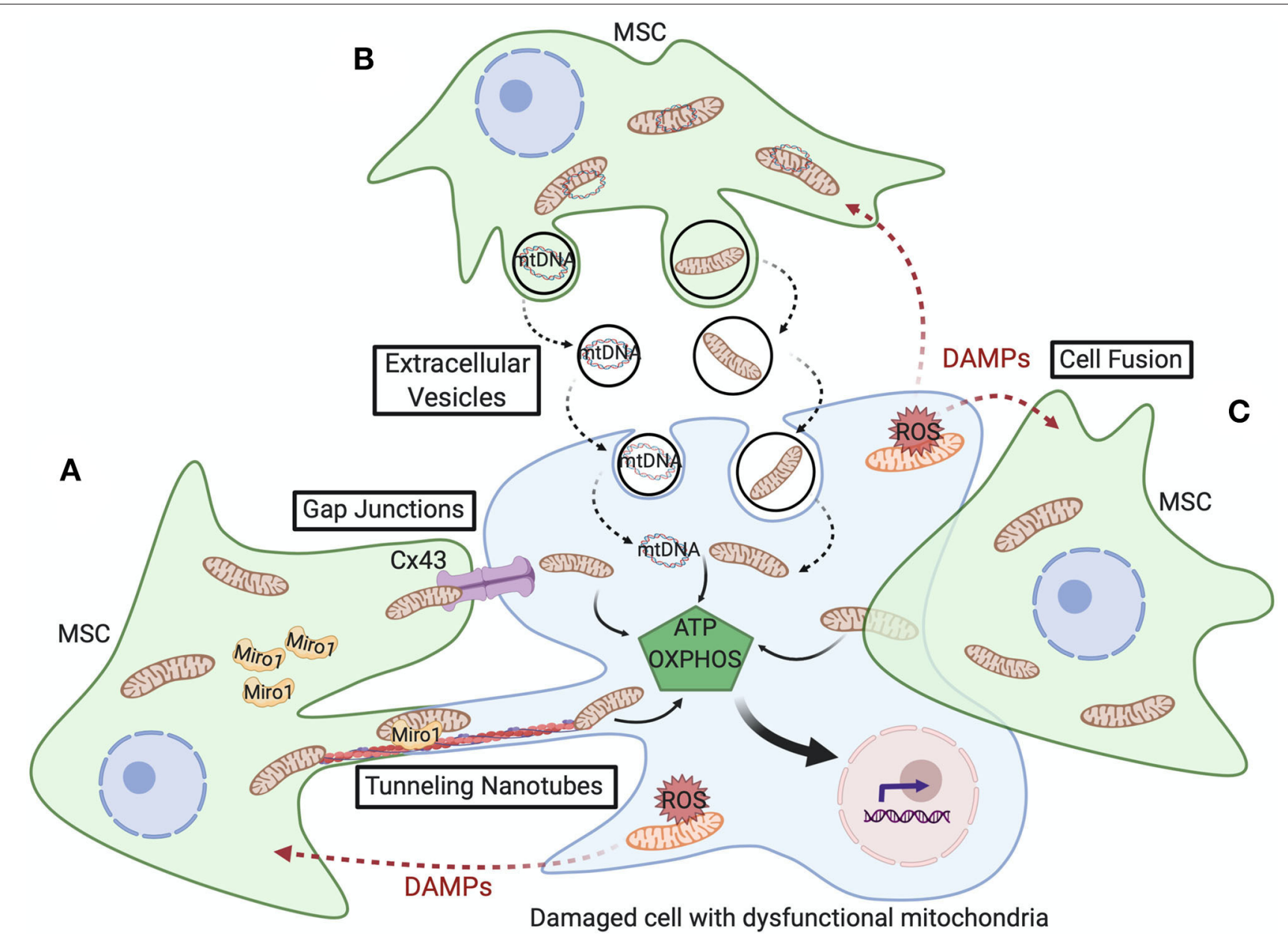

FIGURE 2 | Schematic representation of the various mitochondrial transfer mechanisms utilized between MSCs and damaged cells with dysfunctional mitochondria. (A) Tunneling nanotubes (TNTs) are actin-dependent cytoskeletal protrusions that serve as cytoplasmic bridges between cells. Miro1 regulates transport of mitochondria across TNTs. Cx43-mediated gap junctions also serve at cell-cell junctions to enable mitochondrial transfer. (B) Extracellular vesicles (EVs) can convey mtDNA or fragments of mitochondria and, though less well-documented, macrovesicles are suggested to contain entire mitochondria. (C) Cell fusion enables sharing of cytoplasmic contents during either transient or permanent fusion of the plasma membrane of two cells. Upon transfer of healthy mitochondria from MSCs, respiration increases in the recipient cell and restores cell function, cell survival, and tissue repair.

apoptosis. Masking of PS by annexin V dramatically decreases TNT formation without any significant effect on the quantity of membrane protrusions, indicating that TNT formation by MSCs requires presentation of PS on the membrane protrusions of the injured recipient cell. Other environmental cues and stress conditions, such as hyperglycemia, have also been shown to induce TNT formation in primary cells and cell lines (Lou et al., 2012). In hippocampal astrocytes and neurons, it was proposed that serum starvation and hydrogen peroxide can trigger nanotube formation through p53 activation (Wang et al., 2011). However, using knockout mice and cell lines lacking p53, it has since been shown in murine MSCs and cancer cell lines that TNT development can proceed independently of p 53 , and, therefore, cells must utilize other stress sensing pathways to signal TNT generation (Andresen et al., 2013).

One mechanism for trafficking of mitochondria within TNTs is mediated by motor-adaptor protein complexes related to the mitochondrial Rho GTPase Mirol (encoded by the RHOT1 gene) (Ahmad et al., 2014). Interestingly, mesenchymal cells like smooth muscle cells and fibroblasts express Mirol and can also donate mitochondria, but they do so with low efficiency relative to MSCs (Ahmad et al., 2014). Indeed, Mirol is essential for transport of mitochondria, and its overexpression in MSCs was shown to enhance the transfer of mitochondria alveolar epithelial cells in co-cultures. Mirol overexpression consequently improved rescue of the stressed epithelial cells as measured by ROS accumulation (Ahmad et al., 2014). Indeed, the importance of mitochondrial transfer to the reparative effect of MSCs was also shown in mouse models of lung injury and asthma. MSCs overexpressing Miro1 were more efficient than control MSCs in decreasing airway hyper-responsiveness, decreasing pro-inflammatory cytokines, and restoring ATP levels, and the MSCs did not exhibit changes in their secretory properties or immunomodulatory paracrine signaling. Conversely, Miro1 
knockdown prevented TNT formation and caused the loss of MSC efficacy. In experimental stroke, intravenous administration of MSCs overexpressing Miro1 reversed the effects of ischemia and improved recovery of neurological functions better than unmanipulated MSCs (Babenko et al., 2018). Similarly, induced pluripotent stem cell derived (iPSC)-MSCs intrinsically express a high-level of Mirol and possess higher efficiency of mitochondrial transfer relative to bone marrow MSCs (Zhang et al., 2016). This elevated capacity for mitochondrial trafficking improved MSC efficacy in protection from chemotherapyinduced cardiomyopathy.

A second mechanism for TNT-mediated transport of mitochondria is by gap-junction communication. Connexin 43 (Cx43; encoded by GJA1) is a transmembrane protein which can form hemichannels in association with other connexin proteins to enable direct exchange of ions, metabolites, second messengers, microRNAs, and linear peptides between cells or with the extracellular environment (Ribeiro-Rodrigues et al., 2017). Cx43 also mediates TNT formation for mitochondrial transfer through gap junctions between cells (Islam et al., 2012). In a murine model of LPS-induced lung injury, Islam et al. (2012) showed that $\mathrm{Cx} 43$ expression on both donor bone marrow MSCs and injured alveolar epithelial cells was required for mitochondrial exchange via TNTs. In this model, TNT establishment was initiated from the cell expressing higher levels of $\mathrm{Cx} 43$. Moreover, Cx43 knockdown on MSCs impaired TNT generation and thus these Cx43-deficient MSCs could not rescue the injured epithelial cells (Islam et al., 2012). Further evidence for Cx43 in TNT development in lung injury comes from reports showing that $\mathrm{Cx} 43$ is upregulated in mouse lungs during asthma and that overexpression of $\mathrm{Cx} 43$ in therapeutically administered iPSC-MSCs restores mitochondrial membrane potential, decreases excess mucus secretion, and reduces inflammatory cells and cytokines caused by lung injury (Yao et al., 2015, 2018). Stable knockdown of Cx43 reduced mitochondrial transfer and abolished the benefits of the cellular therapy.

\section{Extracellular Vesicles}

Extracellular vesicles (EVs) are heterogeneous subcellular structures consisting of a lipid bilayer, often containing proteins and receptors, that encapsulates molecular cargo present in the cell of origin (Pitt et al., 2016). Depending on their biogenesis, EVs vary in size, morphology, and the contents they transport. Exosomes are EVs created by endocytosis or reverse budding that range in size from 30 to $150 \mathrm{~nm}$ in diameter. Exosomes engulf small molecules within the cell, including proteins, lipids, carbohydrates, metabolites, and genetic material including small RNAs, mtDNA, mRNA, and microRNAs, and are subsequently secreted into the extracellular space (Van Niel et al., 2018). On the other hand, microvesicles are EVs created from the outward budding of the plasma membrane to form $0.1-1 \mu \mathrm{m}$ diameter structures that are able to carry larger cargo such as organelles. Larger microvesicles created by membrane blebbing, such as apoptotic bodies ranging $1-2 \mu \mathrm{m}$ in diameter and oncosomes $1-10 \mu \mathrm{m}$ in diameter, can also carry mitochondrial components and other metabolic modifiers (Minciacchi et al., 2015; Jiang et al., 2017).

Exosomes and microvesicles are released by diverse cell types including MSCs (Phinney et al., 2015), platelets, endothelial cells, cancer cells (Raposo and Stoorvogel, 2013), and astrocytes (Hayakawa et al., 2016). MSCs can transfer mtDNA and mitochondrial cargo to macrophages and other cells through release of EVs (Islam et al., 2012; Phinney et al., 2015). Studies have also reported transport of mitochondria via both TNTs and EVs from MSCs to different recipient cells, including lung alveolar epithelial cells and macrophages (Islam et al., 2012; Jackson et al., 2016). Compared to TNTs, the mechanisms mediating mitochondrial transfer by EVs are less well-understood. Additionally, evidence suggests that compromised mitochondria might be preferentially exported in this context. Phinney et al. (2015) found that human MSCs target depolarized mitochondria for packaging at the plasma membrane via arrestin domain-containing protein 1mediated microvesicles. The mitochondria containing vesicles are engulfed and reutilized by macrophages. Interestingly, the macrophages are simultaneously subjected to de-sensitization by miRNA-containing exosomes produced by the MSCs that inhibit activation of Toll-like receptor signaling and prevent rejection of the ingested mitochondria. Despite the poor quality of the donor mitochondria, the recipient macrophages demonstrate greater bioenergetic capacity, suggesting mutual benefit to both the MSCs and macrophages.

\section{Cell Fusion}

Cell fusion is a process in which uninuclear cells combine to share organelles and cytosolic components by merging plasma membranes and forming multinuclear cells. Cell fusion can be partial (temporary) or complete (permanent). Partial cell fusion includes a direct transient exchange of protein complexes and subcellular organelles between cells. A complete cell fusion occurs when cells share the entire cytoplasm and acquire a unique permeant karyotype. Cell fusion is predominantly reported to modulate the development of organs during embryogenesis, morphogenesis, and cellular differentiation. Indeed, the placenta, bone, and muscle rely on cell fusion for proper organ function.

Cell fusion is used, albeit rarely, for MSC-driven mitochondria sharing. Underlying mechanisms for mitochondria exchange through cell fusion remain elusive; although, rearrangement of the actin cytoskeleton and fusogenic glycoproteins across the membranes of both cells is required (Aguilar et al., 2013; Shilagardi et al., 2013). Mitochondria transfer has been shown to occur through complete cell fusion after the selective loss of the donor cell's nucleus (Alvarez-Dolado et al., 2003). Acquistapace et al. (2011) reported that mitochondrial transfer via partial cell fusion of human bone marrow MSCs or human multipotent adipose-derived stem cells (hMADS) with mature murine cardiomyocytes could promote reprogramming of the cardiomyocytes to a progenitor-like state. In this process, mtDNA depletion of MSCs did not affect cell fusion capacity, but it did result in a dramatic decrease in their reprogramming efficiency. These data suggest that de-differentiation of the 
TABLE 1 | Summary of mitochondrial transfer between MSCs and recipient cells of different origins.

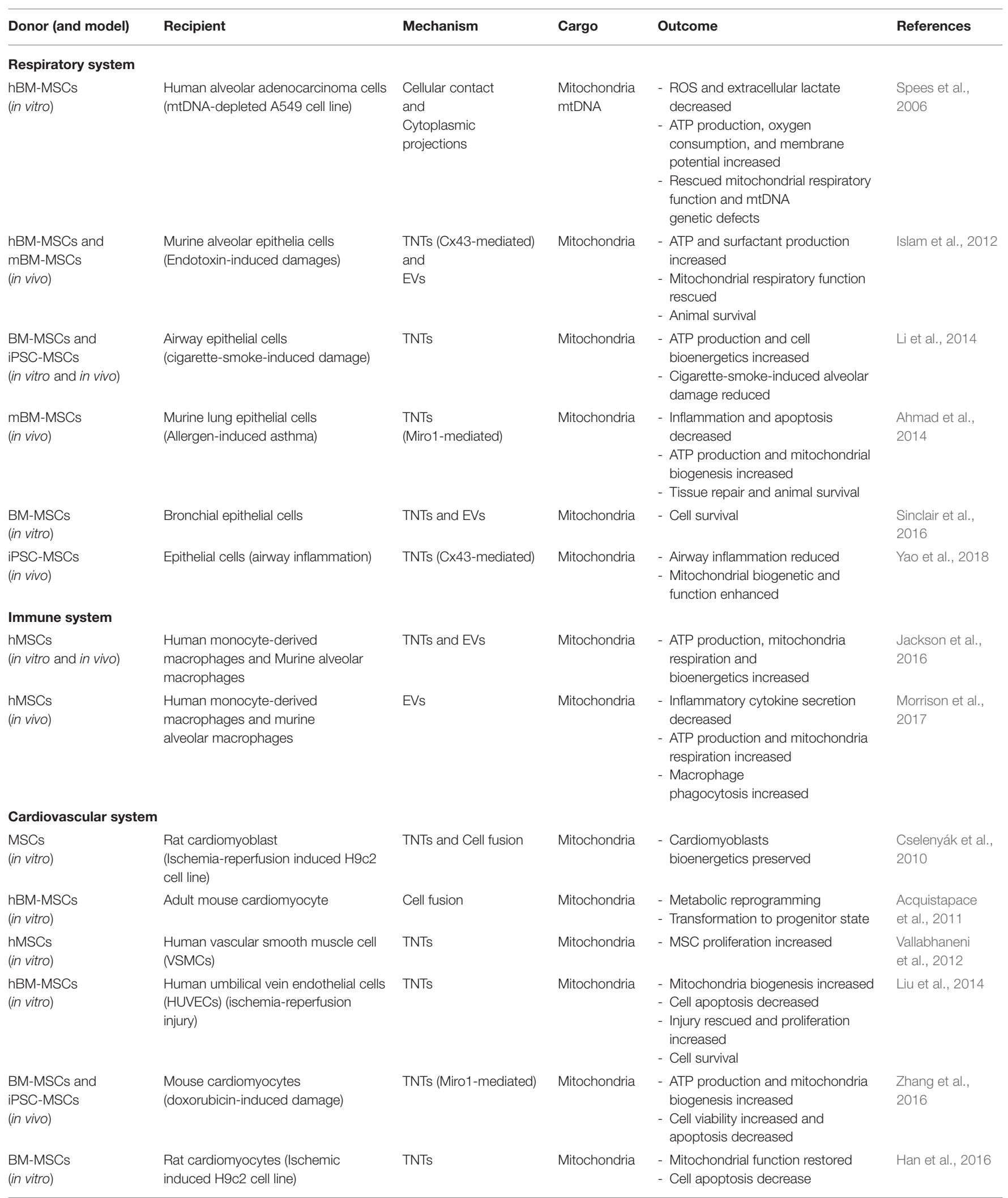


TABLE 1 | Continued

\begin{tabular}{|c|c|c|c|c|c|}
\hline Donor (and model) & Recipient & Mechanism & Cargo & Outcome & References \\
\hline $\begin{array}{l}\text { hMADSs } \\
\text { (in vitro and in vivo) }\end{array}$ & $\begin{array}{l}\text { Cardiomyocytes and endothelial cells } \\
\text { (with oxidative challenge) }\end{array}$ & TNTS & Mitochondria & $\begin{array}{l}\text { - Mitochondrial biogenesis increased } \\
\text { - Cell apoptosis decreased }\end{array}$ & $\begin{array}{l}\text { Mahrouf-Yorgov } \\
\text { et al., } 2017\end{array}$ \\
\hline \multicolumn{6}{|l|}{ Neurological system } \\
\hline $\begin{array}{l}\text { BM-MSCs } \\
\text { (in vitro) }\end{array}$ & Rat cortical neurons (post-stroke) & $\begin{array}{l}\text { Transient cell fusion } \\
\text { (Miro1 upregulated) }\end{array}$ & Mitochondria & $\begin{array}{l}\text { - Restored neurological activity and } \\
\text { cell survival }\end{array}$ & $\begin{array}{l}\text { Babenko et al., } \\
2015\end{array}$ \\
\hline \multicolumn{6}{|l|}{ Renal system } \\
\hline $\begin{array}{l}\text { MSCs } \\
\text { (in vitro) }\end{array}$ & Rat renal tubular cells & TNTS & Mitochondria & $\begin{array}{l}\text { - MSC differentiation into kidney } \\
\text { tubular cells }\end{array}$ & $\begin{array}{l}\text { Plotnikov et al., } \\
2010\end{array}$ \\
\hline \multicolumn{6}{|l|}{ Corneal system } \\
\hline $\begin{array}{l}\text { iPSC-MSCS } \\
\text { (in vitro) }\end{array}$ & Corneal epithelial cells & TNTs (Miro1-mediated) & Mitochondria & $\begin{array}{l}\text { - Mitochondria respiration and } \\
\text { function increased } \\
\text { - Corneal wound healing improved }\end{array}$ & Jiang et al., 2016 \\
\hline
\end{tabular}

BM-MSCs, Bone marrow mesenchymal stromal cells; hBM-MSCs, Human bone marrow mesenchymal stromal cells; mBM-MSCs, Murine bone marrow mesenchymal stromal cells; iPSC-MSCs, pluripotent stem cell-derived mesenchymal stem cells; hMADS, Human multipotent adipose-derived stem cells; TNT, Tunneling nanotubes; EV, Extracellular vesicles.

cardiomyocytes was due to metabolic reprogramming by MSC mitochondria (Acquistapace et al., 2011).

\section{Therapeutic Applications in Mitochondrial Transfer}

Accumulating data implicates mitochondrial donation from MSCs as a critical component of their therapeutic efficacy. Several diseases and organ systems have been examined, including the respiratory, cardiovascular, neurological and renal systems. Table 1 summarizes representative reports from the current literature.

\section{Neurological System}

Several ongoing clinical trials indicate the feasibility and safety of using MSCs for the treatment of neurological injury and degenerative diseases affecting the brain, spinal cord, and visual system. Whereas, some studies implicate the neuroprotective secretome of MSCs in fostering repair and regeneration, others suggest that metabolic rescue is crucial for the therapeutic benefit of MSCs. Babenko et al. (2015) demonstrated that preferential cytosol transfer from neural cells to MSCs could enhance their neuroprotective capacity. This priming from coculture with neurons resulted in doubling of Mirol abundance and elevated production of brain-derived neurotrophic factor in MSCs. Mitochondrial transfer was confirmed from MSCs to neurons and astrocytes in cocultures. Using this priming method in a stroke injury model, authors showed that delivery of neuron-primed MSCs reduced total volume of ischemic lesions and severity of neurological deficits, as measured by response to tactile and proprioceptive stimuli (Babenko et al., 2015). Retinal ischemia caused by stroke can also cause lifelong visual impairments attributed to mitochondrial dysfunction and cell death of the retinal ganglion cells and pigmented epithelium. Nguyen et al. (2019) showed that intravenous transplantation of MSCs restores mitochondrial respiration, morphology, and dynamics in these cells, suggesting that MSC therapy after stroke could also benefit the visual system through mitochondrial transfer. Retinal ganglion cell repair by MSC mitochondrial transfer was also shown in a model of retinal degeneration in which mice lacking the $\mathrm{NADH}$ dehydrogenase Fe-S protein 4 gene $\left(N d u f_{s} 4\right)$ experience deterioration of the nerves of the eyes (Jiang et al., 2019). Following local delivery of human iPSC-MSCs into the vitreous cavity of the eye, successful transfer of mitochondria was confirmed in retinal ganglion cells and their loss by cell death was prevented. Electrical activity measured by electroretinogram also indicated that MSCs protected visual function. Authors confirmed absence of human nuclear DNA in the mouse retina, arguing against permanent cell fusion or direct differentiation of MSCs into neural cell lineages.

\section{Respiratory System}

The therapeutic effect of mitochondrial transfer from MSCs has been demonstrated in respiratory disease by several independent groups using multiple models of lung injury and asthma. Islam et al. (2012) were the first to report that it was mitochondrial transfer from MSCs to pulmonary alveoli that restored alveolar bioenergetics. They showed that intranasal or intratracheal instillation of human or murine MSCs increased animal survival in lipopolysaccharide (LPS)induced acute lung injury. Using live lung microscopy, they observed that mitochondrial transfer occurred through $\mathrm{Cx} 43$ driven TNTs and by EVs in a $\mathrm{Ca}^{2+}$ dependent manner. MSCs reduced leukocyte numbers, normalized surfactant secretion, and improved ATP levels in alveolar epithelia. In an allergeninduced asthma model, Ahmad et al. (2014) demonstrated that MSC efficacy and TNT-mediated mitochondrial transfer from MSCs to injured lung epithelial cells could be amplified by overexpression of Mirol in donor MSCs, while its knockdown reduced therapeutic efficacy. In this study, instillation of MSCs reduced apoptosis in airway epithelium and rescued mitochondrial biogenetics. In a subsequent asthma mouse model study, local transplantation of iPSC-MSCs reduced airway inflammation and improved mitochondrial function in damaged 
epithelial cells through Cx43-dependent TNTs (Yao et al., 2018). Additionally, in a model of chronic obstructive pulmonary disease, mitochondrial transfer from iPSC-MSCs was shown to be more effective than bone marrow MSCs in rescuing cigarette smoke-induced mitochondrial dysfunction, attributed in part to greater mitochondrial transfer efficiency from the pluripotent source of MSCs (Li X. et al., 2018).

\section{Immune System}

The therapeutic properties of MSCs derive in part from their capacity to modulate the innate and adaptive immune systems, including macrophages, dendritic cells, NK cells, and T and B lymphocytes (Weiss and Dahlke, 2019). Ex vivo and in vivo studies have demonstrated that mitochondrial transfer from MSCs to macrophages improves phagocytic activity in the recipient cells (Jackson et al., 2016). Phinney et al. (2015) showed that under oxidative stress, MSCs undergo mitophagy and unload their depolarized mitochondria to macrophages. The EVs engulfed by macrophages augmented macrophage bioenergetics. In a study of acute respiratory distress syndrome, EV-mediated mitochondrial transfer from MSCs to macrophages resulted in repression of proinflammatory cytokine secretion and improved phagocytic capacity by promoting an M2 anti-inflammatory phenotype (Morrison et al., 2017). Collectively, these studies aid our understanding of how metabolic reprogramming can modify the inflammatory profiles of immune cells and complement the direct production of immunomodulatory factors by MSCs.

\section{Cardiovascular System}

Mitochondrial dysfunction is implicated in the development and pathophysiology of heart failure (Zhou and Tian, 2018). As in the lung, mitochondrial transfer in cardiac tissue has been shown to improve outcomes through metabolic reprogramming (Koyanagi et al., 2005; Plotnikov et al., 2008). In an ex vivo ischemic heart disease model, bone marrow MSCs rescued damaged cardiomyocytes through TNTmediated mitochondrial transfer (Han et al., 2016). In this study, mitochondrial membrane potential and function were elevated in the cardiomyocytes and apoptosis was reduced (Han et al., 2016). Further, MSCs with a high efficiency of mitochondrial transfer, iPSC-MSCs expressing Mirol at high intrinsic levels and those with ectopic Mirol expression, were more effective in alleviating injury to cardiac cells in a model of anthracycline-induced cardiomyopathy (Zhang et al., 2016). In co-cultures of bone marrow MSCs and terminally differentiated mouse cardiomyocytes, partial cell fusion and the resulting donation of mitochondria promoted reversion of mature cardiomyocytes to a progenitor-like state (Acquistapace et al., 2011).

Multiple studies have also reported bidirectional trafficking of mitochondria. For example, in co-cultures of human primary MSCs and vascular smooth muscle cells (VSMCs), no preferential direction of mitochondrial movement was observed between the cells (Vallabhaneni et al., 2012). Moreover, the mitochondrial transfer from VSMCs to MSCs promoted proliferation of the recipient MSCs (Vallabhaneni et al., 2012).
Additionally, mtDNA-depleted VSMCs were unable to stimulate MSC proliferation, indicating that functional mitochondria in the donor cells was instrumental in initiating cell cycling. Mahrouf-Yorgov et al. (2017) reported that damage signals released by injured cells can activate anti-apoptotic and regenerative functions of MSCs. In in vitro models of hydrogen peroxide or doxorubicin-induced cellular damage and a model of myocardial infarction, they found that MSCs sense and uptake DAMPs, including dysfunctional mitochondria, that are released from endothelial cells or cardiomyocytes (MahroufYorgov et al., 2017). MSCs engrafted into infarcted hearts upregulated heme oxygenase-1 and increased mitochondrial biogenesis. Conversely, when phagocytosis or heme oxygenase activity was inhibited, engrafted MSCs lost their ability to protect cardiac tissue. Collectively, these data highlight the role that mitochondria play as signaling molecules and the importance of MSC sensing of the microenvironment to trigger therapeutic efficacy.

In summary, mitochondrial transfer plays a central role in metabolic rescue and phenotypic alteration of a variety of organ systems that are responsive to MSC therapy. Multiple lines of evidence support the importance of bi-directional signaling between donor and recipient for activation of MSC trophic and immune regulatory activities. This crosstalk can occur via engulfment of mitochondrial cargo and other DAMPs by MSCs, sharing of cytosolic components, and/or interactions at the plasma membrane. MSCs are then primed to donate mitochondria to neighboring cells in need to assist in restoration of damaged tissue.

\section{MSCS AS MODIFIERS OF CANCER METABOLISM}

These same mechanisms that are beneficial in regenerative medicine can be hijacked in malignancy, whereby transfer of mitochondria and/or mtDNA to cancer cells increases mitochondrial content and enhances OXPHOS to favor proliferation and invasion (Herst et al., 2018). Cancer cell survival, proliferation, and motility often requires adaptation to oxygen-poor conditions and increased nutrient demand. Cancer cells use multiple metabolic strategies to overcome these challenges and obtain the resources they need for ATP production and anabolic processes (Luengo et al., 2017). Some of these strategies include altered expression of metabolic genes or utilization of alternate fuels, but others include exploitation of the metabolic capacities of non-malignant cells associated with the tumor.

MSCs contribute to maintenance of normal tissue homeostasis, but also serve as a reservoir for generation of other stromal derivatives, such as cancer associated fibroblasts (CAFs) and tumor-associated stroma, that alter the biophysical, chemical, and cellular composition of the tumor microenvironment. MSCs recruited to tumors can differentiate into CAFs or remain as multipotent tumorassociated MSCs (Liu et al., 2019). Collectively, the stromal components of the tumor microenvironment have been linked 
to tumorigenesis, angiogenesis, metastasis, immunosuppression, drug resistance, maintenance of cancer stemness, extracellular matrix remodeling, and metabolic reprogramming (Liu et al., 2019). The role of MSCs in tumor initiation, growth, and resistance to treatment is debated, but their ability to modify cancer cell metabolism and the metabolic environment suggests that MSCs are centrally poised to alter malignancy, angiogenesis, and immune cell infiltrates. We detail below the roles that MSCs play in shaping the metabolic landscape in cancer.

\section{Solid Tumors}

MSCs or other MSC derivatives have been shown to donate mitochondria to cell lines from breast cancer, prostate cancer, lung adenocarcinoma, melanoma, glioma, and osteosarcoma (Spees et al., 2006; Schichor et al., 2012; Pasquier et al., 2013; Caicedo et al., 2015; Lin et al., 2015; Dong et al., 2017; Ippolito et al., 2019). The first demonstration of mitochondrial transfer from MSCs was observed during the co-culture of bone marrow MSCs and human A549 lung adenocarcinoma cells depleted of mtDNA by serial passage with ethidium bromide $\left(\rho^{\circ}\right.$ or rho-zero cells) (Spees et al., 2006). Further, it was shown that the respiratory defects of $\rho^{\circ}$ cells are rescued upon receipt of mitochondria or mtDNA from MSCs or even by uptake of isolated mitochondria or mtDNA available in the medium (Spees et al., 2006; Caicedo et al., 2015; Pacak et al., 2015). In two more recent studies, one group showed that $\rho^{\circ}$ B16 mouse melanoma and $\rho^{\circ} 4 \mathrm{~T} 1$ breast carcinoma cells were able to form tumors in vivo only when they acquired mitochondria (and mtDNA) from host cells at the transplant site (Tan et al., 2015; Dong et al., 2017). A fully functional respirasome (CI, CII, and CIV supercomplex) was restored in B16 cells of the primary tumor and in metastases, with evidence of mtDNA from the host shown by sequencing. Indeed, $\rho^{\circ}$ cells recovered from tumors exhibited similar levels of respiration as the parental lines. This was confirmed through measurement of lactate and ATP levels; glucose uptake; and enzymatic activity of succinate dehydrogenase, succinate quinone reductase, and citrate synthase. In independent studies, transfer of mitochondria to in vitro cultured cancer cells has been found to result in elevated levels of OXPHOS and greater tumorigenicity, proliferation, and invasiveness (Caicedo et al., 2015; Lu et al., 2017). Mitochondrial transfer to breast cancer cells was also shown to enhance chemoresistance to doxorubicin (Pasquier et al., 2013). Collectively, these data suggest that mitochondrial transfer from stroma can play a key role in facilitating bioenergetic resilience of cancer cells in vitro and within the tumor microenvironment.

Reports of mitochondrial transfer from MSCs and CAFs to cancer cells point to TNTs as the chief delivery route (Pasquier et al., 2013; Ippolito et al., 2019). Yet, coupling between MSCs and glioma cells has also shown that MSCs share mitochondrial content via $\mathrm{Cx} 43$ gap junctions and cell fusion, which authors described as syncytia because of the extensive structural interactions and functional exchange of cellular contents (Schichor et al., 2012). Future research should aim to determine the effects of metabolic modification of tumors by the stroma on drug resistance mechanisms and immunogenicity of cold tumors (Liu and Curran, 2020).

\section{Hematological Malignancies}

Bone marrow niches, consisting of various cell types including stromal and hematopoietic cells, have critical roles in regulating the fate of adult HSCs in terms of quiescence, self-renewal, and differentiation (Méndez-Ferrer et al., 2020). These niches maintain hematopoietic homeostasis through soluble factors, direct cell-cell contact, and cell-surface ligands. Indeed, MSCs directly interact with HSCs and secrete hematopoietic cytokines and growth factors that dictate HSC cell cycle entry and lineage maturation (Saleh et al., 2015). In hematological malignancies, remodeling of bone marrow niches has been found to create an environment that supports malignant stem cells and impairs maintenance of normal HSCs (Pievani et al., 2020). Crosstalk with cells in the bone marrow microenvironment can also contribute to drug resistance and relapse via modification of gradients of soluble factors/metabolites, dysregulation of extracellular matrix deposition, and by direct cell-cell interactions, many of which alter cancer cell metabolism (Konopleva et al., 2002; Samudio et al., 2008). Cell intrinsic mutations can also drive these metabolic adaptations (Faubert et al., 2020). Moreover, it is clear that these strategies of adaptation to nutrients in the environment and metabolic reprogramming are frequently employed by hematological malignancies to support aggressive anabolic growth and chemoresistance (Basak and Banerjee, 2015). Table 2 summarizes reports of mitochondrial transfer in hematological malignancies.

\section{Acute Myeloid Leukemia}

Substantial evidence points to the bone marrow microenvironment as a major contributor to the pathogenesis of leukemia, including adaptations in energy metabolism that confer resistance to chemotherapy. Bone marrow MSCs have been shown to drive reprogramming of acute myeloid leukemia (AML) metabolism toward aerobic glycolysis thereby promoting the Warburg effect by release of paracrine effectors (Samudio et al., 2008). In this study, MSCs induced depolarization of the mitochondria of AML cells by upregulation of uncoupling protein 2 (UCP2), leading to decreased production of ROS and reduced susceptibility to chemotherapeutic drugs, including mitoxantrone, cytosine arabinoside, and vincristine. Another mechanism proposed to protect AML cells from toxic ROS accumulation is export of superoxides to MSCs through gap junctions, as has been demonstrated to protect HSCs in the aging niche (Ishikawa et al., 2012).

As observed in solid tumors, mitochondrial transfer from MSCs is also reported in hematological malignancies, including AML. Primary and cultured AML cells readily uptake mitochondria from bone marrow stromal cells both in vitro and in vivo in xenotransplantation models (Moschoi et al., 2016; Marlein et al., 2017). Compared to HSCs, AML cells contain more mtDNA, larger numbers of mitochondria, and depend heavily on OXPHOS (Boultwood et al., 1996; Škrtić et al., 2011). Bone marrow stroma serve as an important reservoir of mitochondria for AML cells, as elevated OXPHOS can only be maintained in culture with MSCs containing functional mitochondria. AML cells cultured with $\rho^{\circ}$ MS-5 stromal cells or separated from MS-5 cells by transwell membranes fail to maintain high 
TABLE 2 | Summary of mitochondria transfer between MSCs and recipient cells of hematological malignancies.

\begin{tabular}{|c|c|c|c|c|}
\hline Recipient & Model & Mechanism & Outcome & References \\
\hline
\end{tabular}

\section{Acute myeloid leukemia (AML)}

Primary AML blasts and AML cell lines: HL-60, Kasumi-1, KG-1, and U-937

Primary AML blasts

\section{Acute lymphocytic leukemia (ALL)}

Primary B-ALL blasts and BCP-ALL cell lines: NALM6 (B-Other) and REH (TEL-AML1)

Primary T-ALL cells and T-ALL cell line: Jurkat MOLM-14, NB-4, SKM-1, THP-1,
In vitro coculture

In vitro coculture
In vitro coculture

and

NSG xenograft

model

In vitro coculture

and

NSG xenograft

model
TNT-mediated and endocytosis-dependent mitochondrial transfer to AML cells

TNT-mediated mitochondrial transfer to AML cells upon NOX2 upregulation and ROS enhancement
Moschoi et al., 2016

Increased tumorigenic potential and chemoresistance

- Extracellular lactate production reduced

Marlein et al., 2017

- ATP level, oxygen consumption and mitochondrial membrane potential increased

- NOX2 inhibition blocked mitochondrial transfer, increased AML cell apoptosis, and improved animal survival

in xenotransplantation

TNT-mediated mitochondrial transfer to BCP-ALL cells

- Increased tumorigenic potential and chemoresistance

Primary T-ALL cells and T-ALL cell line: Jurkat increased in T-ALL cells
Not described

TNT-mediated mitochondrial transfer to BCP-ALL cells

and NSG xenograft model

In vitro coculture
Bidirectional TNT-mediated mitochondrial transfer modulated by adhesion molecule ICAM-1

TNT-mediated mitochondrial transfer mediated by CD38
- Glucose uptake and lactate production

- Mitochondrial membrane potential and ROS level decreased

- Promoted a pro-glycolytic shift in T-ALL cells

- Mitochondrial fission governed by ERK-mediated phosphorylation of Drp1

- T-ALL cell chemoresistance

- MSC acquisition of cancer-associated fibroblast phenotype

- BCP-ALL cell chemoresistance

- Tumor progression in animals

- Microtubule inhibitors interrupted mitochondrial trafficking and reduced drug resistance

- T-ALL cells transfer more mitochondria to MSCs while receive fewer

- T-ALL cells chemoresistance

- Blockage of mitochondria transfer by inhibiting TNT formation decreased the drug cytotoxicity of T-ALL cells

In vitro coculture
and
NSG xenograft
model

In vitro coculture model

\author{
- ATP level and OXPHOS increased in MM cells Marlein et al., 2019 \\ - Chemotherapy drugs increased \\ mitochondrial transfer and MM cell \\ tumorgenicity \\ - CD38 knockdown blocked mitochondrial \\ transfer and improved animal survival \\ (1)
}

Polak et al., 2015

Cai et al., 2016

Burt et al., 2019

Wang et al., 2018 levels of mitochondrial ATP production (Moschoi et al., 2016). Marlein et al. (2017) showed that AML initiates mitochondrial transfer with MSCs by NADPH oxidase-2 (NOX2)-dependent superoxide production. This mechanism not only improves bioenergetics in AML cells, but also increases chemoresistance to cytarabine (Moschoi et al., 2016). In this way, AML cells exploit oxidative stress to acquire greater mitochondrial capacity and chemoresistance. Indeed, some commonly used chemotherapeutic agents amplify mitochondrial transfer to AML, including cytarabine, etoposide, and doxorubicin (Moschoi et al., 2016). Thus, targeting mitochondria and/or mitochondrial transfer could have therapeutic advantages in chemotherapy-resistant AML (Lagadinou et al., 2013). For example, cytarabine residual AML cells (cytarabine resistant) utilize high levels of OXPHOS and thus agents that inhibit mitochondrial protein synthesis, electron transfer, or fatty-acid oxidation have the greatest anti-leukemic effects in this resistant population (Farge et al., 2017).

Several of the approaches used to disrupt mitochondrial transfer are aimed at blocking TNTs, endocytosis, or superoxides that can prime MSCs. The CD38 surface marker has been implicated in TNT formation and mitochondrial transfer from MSCs to AML, but also can be leveraged for targeting AML cells for phagocytosis by anti-CD38 antibodies (Farber et al., 2018; Mistry et al., 2019). Daratumumab is the first of several antiCD38 monoclonal antibody-based therapeutics that improve 
anti-tumor immunity and also demonstrate an inhibitory effect on AML mitochondrial transfer in vitro and in vivo (Farber et al., 2018; Mistry et al., 2019). Master regulators of mitochondrial biogenesis and activity including PGC-1 $\alpha$ and NOX2 are also promising targets. AML blasts stimulate MSCs to produce more mitochondria, consume more oxygen, and activate PGC- $1 \alpha$ (Marlein et al., 2018). PGC-1 $\alpha$ was important for mitochondrial transfer to AML cells, and authors showed that inactivation of PGC- $1 \alpha$ by knockdown or by reduction in superoxide levels with $\mathrm{N}$-acetylcysteine impaired mitochondrial transfer (Marlein et al., 2017, 2018). In an NSG xenograft model, PGC-1 $\alpha$ deficient bone marrow stromal cells co-transplanted subcutaneously with AML cells produced smaller tumors relative to control stroma (Marlein et al., 2018). Similarly, inhibition of NOX2 in NSG mice increased AML apoptosis and improved animal survival by preventing mitochondrial transfer (Marlein et al., 2017). Together, these studies highlight the importance of not only exploiting metabolic dependencies of the tumor cell but also understanding how the cells within the microenvironment enable bioenergetic resilience and chemoresistance of the leukemia cells.

\section{Acute Lymphoblastic Leukemia}

Acute lymphoblastic leukemia (ALL) is another hematological malignancy that disrupts normal HSC and progenitor niches as they overtake the local microenvironment of the bone marrow (Colmone et al., 2008). TNT-mediated mitochondrial transfer between B-cell precursor ALL (BCP-ALL) cells and surrounding MSCs has been reported to be a mechanism by which ALL cells regulate release of cytokines and chemokines from the cells that comprise the HSC niche (Polak et al., 2015). Authors showed that BCP-ALL cells use TNTs to signal to primary MSCs. This communication stimulates modification of niche factors, including pro-survival cytokines and chemokines, that promote BCP-ALL survival and chemotherapy resistance. Importantly, disruption of TNTs resensitizes BCP-ALL to treatment. Burt et al. (2019) also suggest that chemotherapy resistance and relapse in ALL arises not from a specific mutation in ALL cells but rather from sheltering of ALL cells by a niche in the bone marrow that protects from the toxic ROS accumulation that many chemotherapeutics rely upon for killing. They made the novel observation that MSCs isolated from the bone marrow of patients treated for ALL with standard induction chemotherapy and rituximab had an abundance of activated MSCs with a CAF-like phenotype. To test whether MSC activation provided protection for ALL cells from chemosensitivity, they modeled MSC activation in vitro with ROS-inducing agents and observed that ALL cells experienced significantly less cell death when able to engage in direct cell-cell contact with MSCs. They further found that toxic levels of intracellular ROS were reduced in ALL cells exposed to chemotherapy, which was mediated by TNTbased communication and transfer of mitochondria from MSCs. Blocking mitochondrial transfer by mitochondrial depletion of MSCs, microtubule inhibitors, or co-culture in transwells impaired ALL cell survival. In another study, bidirectional TNT-mediated mitochondrial transfer between $\mathrm{T}$ cell acute lymphoblastic leukemia (T-ALL) cells and surrounding MSCs was also found to contribute to chemoresistance of this hematological malignancy (Wang et al., 2018). During this interaction, which was mediated by the adhesion molecule ICAM-1, T-ALL cells transferred more mitochondria to MSCs while receiving fewer from MSCs (Wang et al., 2018). In this model, increased mitochondria transfer from T-ALL cells to MSCs was reported as the mechanism employed by T-ALL cells to survive the chemotherapy-induced intracellular ROS (Wang et al., 2018). The preference for export of mitochondria from TALL cells was suggested to be due to a bias toward glycolytic ATP production in contrast to reliance on OXPHOS in AML (Suganuma et al., 2010). Nevertheless, these reports suggest that blocking the route of mitochondria trafficking between MSCs and hematological malignancies is an important area for future research that could have promising therapeutic potential in relapsed or refractory tumors.

\section{Multiple Myeloma}

Similar to T-ALL, heavy reliance on glycolysis is observed in a large subset of patients with multiple myeloma (MM) (Borsi et al., 2014; Dalva-Aydemir et al., 2015). Yet, inhibition of glucose metabolism can lead to compensatory engagement of glutamine oxidation that enables survival and chemoresistance of MM cells (Adekola et al., 2015; Marlein et al., 2019). Marlein et al. (2019) reports that mitochondrial transfer from MSCs to MM cells via tumor-derived TNTs promotes this metabolic switch to OXPHOS. Inhibition of endocytosis by dansylcadavarine had no impact on mitochondrial transfer; whereas, the inhibitor of actin polymerization cytochalasin B significantly reduced mitochondrial transfer. TNT formation was observed in $\sim 60 \%$ of $\mathrm{MM}$ cells in co-culture with bone marrow MSCs, and residual lipid membrane from MM cells was prevalent on MSCs, suggesting that MM readily establish TNTs with MSCs. Mitochondrial trafficking was prevented by a blocking antibody against or knockdown of the surface molecule CD38, which also reduced tumor burden and improved animal survival. Agents targeting CD38 have attracted attention for their inhibitory effect on mitochondrial trafficking between hematologic cancers and MSCs. For example, treatment of relapsed or refractory $\mathrm{MM}$ by daratumumab or isatuximab show marked improvements in overall response rate and progression-free survival (Krejcik et al., 2016; Martin et al., 2019; Moreno et al., 2019). Mitochondrial transfer therefore should be considered part of the malignant phenotype of $\mathrm{MM}$ and one that serves to facilitate chemoresistance. Altogether, targeting TNT-mediated communication between hematologic malignancies and their supportive niche offers a promising approach to overcome drug resistance and relapse.

\section{CONCLUSIONS}

Mitochondria and regulators of mitochondrial activity have emerged as critical determinants of MSC differentiation and function. The contribution of MSCs to homeostasis of hematopoietic stem and progenitor cells within the bone marrow is well-accepted. Yet, as clinical applications for MSC therapies expand, we are now learning that a critical component 
of their regenerative and tropic effects includes donation of their mitochondria to damaged cells. This same altruistic behavior of MSCs can also exacerbate cancer progression and confer chemoresistance to solid and hematological cancers. Relapse after chemotherapy continues to be a major challenge, thus demand for new therapeutic strategies that explore interruption of mitochondrial transfer and metabolic regulation by MSCs is high. Given that intercellular mitochondrial shuttling has broad implications for treatment of injury and disease, further investigation of the mechanisms that regulate MSC priming and mitochondrial transfer should be a future priority.

\section{REFERENCES}

Acquistapace, A., Bru, T., Lesault, P. F., Figeac, F., Coudert, A. E., Le Coz, O., et al. (2011). Human mesenchymal stem cells reprogram adult cardiomyocytes toward a progenitor-like state through partial cell fusion and mitochondria transfer. Stem Cells 29, 812-824. doi: 10.1002/stem.632

Adekola, K. U. A., Dalva Aydemir, S., Ma, S., Zhou, Z., Rosen, S. T., and Shanmugam, M. (2015). Investigating and targeting chronic lymphocytic leukemia metabolism with the human immunodeficiency virus protease inhibitor ritonavir and metformin. Leuk. Lymphoma 56, 450-459. doi: 10.3109/10428194.2014.922180

Aguilar, P. S., Baylies, M. K., Fleissner, A., Helming, L., Inoue, N., Podbilewicz, B., et al. (2013). Genetic basis of cell-cell fusion mechanisms. Trends Genet. 29, 427-437. doi: 10.1016/j.tig.2013.01.011

Ahmad, T., Mukherjee, S., Pattnaik, B., Kumar, M., Singh, S., Rehman, R., et al. (2014). Miro1 regulates intercellular mitochondrial transport and enhances mesenchymal stem cell rescue efficacy. EMBO J. 33, 994-1010. doi: 10.1002/embj.201386030

Akyurekli, C., Le, Y., Richardson, R. B., Fergusson, D., Tay, J., and Allan, D. S. (2015). A systematic review of preclinical studies on the therapeutic potential of mesenchymal stromal cell-derived microvesicles. Stem Cell Rev. Rep. 11, 150-160. doi: 10.1007/s12015-014-9545-9

Almeida, M., and O'Brien, C. A. (2013). Basic biology of skeletal aging: role of stress response pathways. J. Gerontol. Ser. A Biol. Sci. Med. Sci. 68, 1197-1208. doi: 10.1093/gerona/glt079

Alvarez-Dolado, M., Pardal, R., Garcia-Verdugo, J. M., Fike, J. R., Lee, H. O., Pfeffer, K., et al. (2003). Fusion of bone-marrow-derived cells with Purkinje neurons, cardiomyocytes and hepatocytes. Nature 425, 968-973. doi: 10.1038/nature02069

An, J. H., Yang, J. Y., Ahn, B. Y., Cho, S. W., Jung, J. Y., Cho, H. Y., et al. (2010). Enhanced mitochondrial biogenesis contributes to Wnt induced osteoblastic differentiation of C3H10T1/2 cells. Bone 47, 140-150. doi: 10.1016/j.bone.2010.04.593

Andresen, V., Wang, X., Ghimire, S., Omsland, M., Gjertsen, B. T., and Gerdes, H. H. (2013). Tunneling nanotube (TNT) formation is independent of p53 expression. Cell Death Differ. 20:1124. doi: 10.1038/cdd.2013.61

Araldi, E., and Schipani, E. (2010). Hypoxia, HIFs and bone development. Bone 47, 190-196. doi: 10.1016/j.bone.2010.04.606

Asada, N., Takeishi, S., and Frenette, P. S. (2017). Complexity of bone marrow hematopoietic stem cell niche. Int. J. Hematol. 106, 45-54. doi: 10.1007/s12185-017-2262-9

Babenko, V. A., Silachev, D. N., Popkov, V. A., Zorova, L. D., Pevzner, I. B., Plotnikov, E. Y., et al. (2018). Mirol enhances mitochondria transfer from multipotent mesenchymal stem cells (MMSC) to neural cells and improves the efficacy of cell recovery. Molecules 23, 1-14. doi: 10.3390/molecules23 030687

Babenko, V. A., Silachev, D. N., Zorova, L. D., Pevzner, I. B., Khutornenko, A. A., Plotnikov, E. Y., et al. (2015). Improving the post-stroke therapeutic potency of mesenchymal multipotent stromal cells by cocultivation with cortical neurons: the role of crosstalk between cells. Stem Cells Transl. Med. 4, 1011-1020. doi: $10.5966 /$ sctm.2015-0010

\section{AUTHOR CONTRIBUTIONS}

AM and PW contributed to conception and design of the review outlines. AM prepared the figures and tables. All authors contributed to writing sections of the text, revision, reading, and approval of the submitted manuscript.

\section{FUNDING}

This work was supported by grants from the American Society of Hematology Scholar Award and National Institutes of Health (R01DK111599) to PW.

Bahat, A., and Gross, A. (2019). Mitochondrial plasticity in cell fate regulation. J. Biol. Chem. 294, 13852-13863. doi: 10.1074/jbc.REV118.000828

Basak, N. P., and Banerjee, S. (2015). Mitochondrial dependency in progression of acute myeloid leukemia. Mitochondrion 21, 41-48. doi: 10.1016/j.mito.2015.01.006

Bi, S., Liu, Z., Wu, Z., Wang, Z., Liu, X., Wang, S., et al. (2020). SIRT7 antagonizes human stem cell aging as a heterochromatin stabilizer. Protein Cell 11, 483-504. doi: 10.1007/s13238-020-00728-4

Boroumand, P., and Klip, A. (2020). Bone marrow adipose cellscellular interactions and changes with obesity. J. Cell Sci. 133, 1-8. doi: $10.1242 /$ jcs. 238394

Borsi, E., Perrone, G., Terragna, C., Martello, M., Dico, A. F., Solaini, G., et al. (2014). Hypoxia inducible factor-1 alpha as a therapeutic target in multiple myeloma. Oncotarget 5, 1779-1792. doi: 10.18632/oncotarget.1736

Boultwood, J., Fidler, C., Mills, K. I., Frodsham, P. M., Kusec, R., Gaiger, A., et al. (1996). Amplification of mitochondrial DNA in acute myeloid leukaemia. Br. J. Haematol. 95, 426-431. doi: 10.1046/j.1365-2141.1996.d01-1922.x

Boyette, L. B., Creasey, O. A., Guzik, L., Lozito, T., and Tuan, R. S. (2014). Human bone marrow-derived mesenchymal stem cells display enhanced clonogenicity but impaired differentiation with hypoxic preconditioning. Stem Cells Transl. Med. 3, 241-254. doi: 10.5966/sctm.2013-0079

Bukoreshtliev, N. V., Wang, X., Hodneland, E., Gurke, S., Barroso, J. F. V., and Gerdes, H. H. (2009). Selective block of tunneling nanotube (TNT) formation inhibits intercellular organelle transfer between PC12 cells. FEBS Lett. 583, 1481-1488. doi: 10.1016/j.febslet.2009.03.065

Burt, R., Dey, A., Aref, S., Aguiar, M., Akarca, A., Bailey, K., et al. (2019). Activated stromal cells transfer mitochondria to rescue acute lymphoblastic leukemia cells from oxidative stress. Blood 134, 1415-1429. doi: 10.1182/blood.2019001398

Cai, J., Wang, J., Huang, Y., Wu, H., Xia, T., Xiao, J., et al. (2016). ERK/Drp1dependent mitochondrial fission is involved in the MSC-induced drug resistance of T-cell acute lymphoblastic leukemia cells. Cell Death Dis. 7, e2459-e2411. doi: 10.1038/cddis.2016.370

Caicedo, A., Fritz, V., Brondello, J. M., Ayala, M., Dennemont, I., Abdellaoui, N., et al. (2015). MitoCeption as a new tool to assess the effects of mesenchymal stem/stromal cell mitochondria on cancer cell metabolism and function. Sci. Rep. 5, 1-10. doi: 10.1038/srep09073

Carrico, C., Meyer, J. G., He, W., Gibson, B. W., and Verdin, E. (2018). The mitochondrial acylome emerges: proteomics, regulation by sirtuins, and metabolic and disease implications. Cell Metab. 27, 497-512. doi: 10.1016/j.cmet.2018.01.016

Chakrabarti, R., Ji, W. K., Stan, R. V., Sanz, J., de, J., Ryan, T. A., et al. (2018). INF2mediated actin polymerization at the ER stimulates mitochondrial calcium uptake, inner membrane constriction, and division. J. Cell Biol. 217, 251-268. doi: $10.1083 /$ jcb.201709111

Chen, C.-T., Shih, Y.-R. V., Kuo, T. K., Lee, O. K., and Wei, Y.-H. (2008) Coordinated changes of mitochondrial biogenesis and antioxidant enzymes during osteogenic differentiation of human mesenchymal stem cells. Stem Cells 26, 960-968. doi: 10.1634/stemcells.2007-0509

Chen, Q., Hao, W., Xiao, C., Wang, R., Xu, X., Lu, H., et al. (2017). SIRT6 is essential for adipocyte differentiation by regulating mitotic clonal expansion. Cell Rep. 18, 3155-3166. doi: 10.1016/j.celrep.2017.03.006 
Cioffi, M., Vallespinos-Serrano, M., Trabulo, S. M., Fernandez-Marcos, P. J., Firment, A. N., Vazquez, B. N., et al. (2015). MiR-93 controls adiposity via inhibition of Sirt7 and Tbx3. Cell Rep. 12, 1594-1605. doi: 10.1016/j.celrep.2015.08.006

Cipolat, S., De Brito, O. M., Dal Zilio, B., and Scorrano, L. (2004). OPA1 requires mitofusin 1 to promote mitochondrial fusion. Proc. Natl. Acad. Sci. U.S.A. 101, 15927-15932. doi: 10.1073/pnas.0407043101

Cogliati, S., Enriquez, J. A., and Scorrano, L. (2016). Mitochondrial cristae: where beauty meets functionality. Trends Biochem. Sci. 41, 261-273. doi: 10.1016/j.tibs.2016.01.001

Cogliati, S., Frezza, C., Soriano, M. E., Varanita, T., Quintana-Cabrera, R., Corrado, M., et al. (2013). Mitochondrial cristae shape determines respiratory chain supercomplexes assembly and respiratory efficiency. Cell 155, 160-171. doi: 10.1016/j.cell.2013.08.032

Collin, F. (2019). Chemical basis of reactive oxygen species reactivity and involvement in neurodegenerative diseases. Int. J. Mol. Sci. 20. doi: 10.3390/ijms20102407

Colmone, A., Amorim, M., Pontier, A. L., Wang, S., Jablonski, E., and Sipkins, D. A. (2008). Leukemic cells create bone marrow niches that disrupt the behavior of normal hematopoietic progenitor cells. Science (80-.). 322, 1861-1865. doi: 10.1126/science.1164390

Cselenyák, A., Pankotai, E., Horváth, E. M., Kiss, L., and Lacza, Z. (2010). Mesenchymal stem cells rescue cardiomyoblasts from cell death in an in vitro ischemia model via direct cell-to-cell connections. BMC Cell Biol. 11:29. doi: 10.1186/1471-2121-11-29

Dalva-Aydemir, S., Bajpai, R., Martinez, M., Adekola, K. U. A., Kandela, I., Wei, C., et al. (2015). Targeting the metabolic plasticity of multiple myeloma with FDA-approved ritonavir and metformin. Clin. Cancer Res. 21, 1161-1171. doi: 10.1158/1078-0432.CCR-14-1088

Davis, D. M., and Sowinski, S. (2008). Membrane nanotubes: dynamic longdistance connections between animal cells. Nat. Rev. Mol. Cell Biol. 9, 431-436. doi: $10.1038 / \mathrm{nrm} 2399$

De Meester, C., Timmermans, A. D., Balteau, M., Ginion, A., Roelants, V., Noppe, G., et al. (2014). Role of AMP-activated protein kinase in regulating hypoxic survival and proliferation of mesenchymal stem cells. Cardiovasc. Res. 101, 20-29. doi: 10.1093/cvr/cvt227

Deng, R., Liu, Y., He, H., Zhang, H., Zhao, C., Cui, Z., et al. (2020). Haemin pre-treatment augments the cardiac protection of mesenchymal stem cells by inhibiting mitochondrial fission and improving survival. J. Cell. Mol. Med. 24, 431-440. doi: 10.1111/jcmm.14747

Ding, Y., Yang, H., Wang, Y., Chen, J., Ji, Z., and Sun, H. (2017). Sirtuin 3 is required for osteogenic differentiation through maintenance of PGC-1 $\alpha$ SOD2-mediated regulation of mitochondrial function. Int. J. Biol. Sci. 13, 254-264. doi: 10.7150/ijbs.17053

Dong, L. F., Kovarova, J., Bajzikova, M., Bezawork-Geleta, A., Svec, D., Endaya, B., et al. (2017). Horizontal transfer of whole mitochondria restores tumorigenic potential in mitochondrial DNA-deficient cancer cells. elife 6, 1-22. doi: 10.7554/eLife.22187

Du, J., Zhou, Y., Su, X., Yu, J. J., Khan, S., Jiang, H., et al. (2011). Sirt5 is a NAD-dependent protein lysine demalonylase and desuccinylase. Science 334, 806-809. doi: 10.1126/science. 1207861

Ejtehadifar, M., Shamsasenjan, K., Movassaghpour, A., Akbarzadehlaleh, P., Dehdilani, N., Abbasi, P., et al. (2015). The effect of hypoxia on mesenchymal stem cell biology. Adv. Pharm. Bull. 5, 141-149. doi: 10.15171/apb.2 015.021

Ekstrand, M. I., Falkenberg, M., Rantanen, A., Park, C. B., Gaspari, M., Hultenby, K., et al. (2004). Mitochondrial transcription factor A regulates mtDNA copy number in mammals. Hum. Mol. Genet. 13, 935-944. doi: 10.1093/hmg/ ddh 109

Eslaminejad, M. B., Fani, N., and Shahhoseini, M. (2013). Epigenetic regulation of osteogenic and chondrogenic differentiation of mesenchymal stem cells in culture. Cell J. 15, 1-10.

Farber, M., Arnold, L., Chen, Y., Möllmann, M., Duehrsen, U., and Hanoun, M. (2018). Inhibition of CD38 shows anti-leukemic activity in acute myeloid leukemia. Blood 132, 1456-1456. doi: 10.1182/blood-2018-99-113287

Farge, T., Saland, E., de Toni, F., Aroua, N., Hosseini, M., Perry, R., et al. (2017). Chemotherapy-resistant human acute myeloid leukemia cells are not enriched for leukemic stem cells but require oxidative metabolism. Cancer Discov. 7, 716-735. doi: 10.1158/2159-8290.CD-16-0441

Faubert, B., Solmonson, A., and DeBerardinis, R. J. (2020). Metabolic reprogramming and cancer progression. Science 368:eaaw5473. doi: $10.1126 /$ science.aaw5473

Fernandez-Marcos, P. J., and Auwerx, J. (2011). Regulation of PGC-1 $\alpha$, a nodal regulator of mitochondrial biogenesis. Am. J. Clin. Nutr. 93, 884S-890S. doi: 10.3945/ajen.110.001917

Forni, M. F., Peloggia, J., Trudeau, K., Shirihai, O., and Kowaltowski, A. J. (2016). Murine mesenchymal stem cell commitment to differentiation is regulated by mitochondrial dynamics. Stem Cells 34, 743-755. doi: 10.1002/stem.2248

Garg, A. D., Nowis, D., Golab, J., Vandenabeele, P., Krysko, D. V., and Agostinis, P. (2010). Immunogenic cell death, DAMPs and anticancer therapeutics: an emerging amalgamation. Biochim. Biophys. Acta Rev. Cancer 1805, 53-71. doi: 10.1016/j.bbcan.2009.08.003

Geißler, S., Textor, M., Kühnisch, J., Könnig, D., Klein, O., Ode, A., et al. (2012). Functional comparison of chronological and in vitro aging: differential role of the cytoskeleton and mitochondria in mesenchymal stromal cells. PLoS ONE 7:e52700. doi: 10.1371/journal.pone.0052700

Gerdes, H. H., Bukoreshtliev, N. V., and Barroso, J. F. V. (2007). Tunneling nanotubes: a new route for the exchange of components between animal cells. FEBS Lett. 581, 2194-2201. doi: 10.1016/j.febslet.2007.03.071

Gilkerson, R. W., Selker, J. M. L., and Capaldi, R. A. (2003). The cristal membrane of mitochondria is the principal site of oxidative phosphorylation. FEBS Lett. 546, 355-358. doi: 10.1016/S0014-5793(03)00633-1

Grayson, W. L., Zhao, F., Izadpanah, R., Bunnell, B., and Ma, T. (2006). Effects of hypoxia on human mesenchymal stem cell expansion and plasticity in $3 \mathrm{D}$ constructs. J. Cell. Physiol. 207, 331-339. doi: 10.1002/jcp.20571

Griessinger, E., Moschoi, R., Biondani, G., and Peyron, J. F. (2017). Mitochondrial transfer in the leukemia microenvironment. Trends Cancer 3, 828-839. doi: 10.1016/j.trecan.2017.10.003

Gurke, S., Barroso, J. F. V., and Gerdes, H. H. (2008). The art of cellular communication: tunneling nanotubes bridge the divide. Histochem. Cell Biol. 129, 539-550. doi: 10.1007/s00418-008-0412-0

Han, H., Hu, J., Yan, Q., Zhu, J., Zhu, Z., Chen, Y., et al. (2016). Bone marrow-derived mesenchymal stem cells rescue injured $\mathrm{H} 9 \mathrm{c} 2$ cells via transferring intact mitochondria through tunneling nanotubes in an in vitro simulated ischemia/reperfusion model. Mol. Med. Rep. 13, 1517-1524. doi: $10.3892 / \mathrm{mmr} .2015 .4726$

Hatch, A. L., Gurel, P. S., and Higgs, H. N. (2014). Novel roles for actin in mitochondrial fission. J. Cell Sci. 127, 4549-4560. doi: 10.1242/jcs.153791

Hayakawa, K., Esposito, E., Wang, X., Terasaki, Y., Liu, Y., Xing, C., et al. (2016). Transfer of mitochondria from astrocytes to neurons after stroke. Nature 535, 551-555. doi: 10.1038/nature18928

He, K., Shi, X., Zhang, X., Dang, S., Ma, X., Liu, F., et al. (2011). Long-distance intercellular connectivity between cardiomyocytes and cardiofibroblasts mediated by membrane nanotubes. Cardiovasc. Res. 92, 39-47. doi: $10.1093 / \mathrm{cvr} / \mathrm{cvr} 189$

Herst, P. M., Dawson, R. H., and Berridge, M. V. (2018). Intercellular communication in tumor biology: a role for mitochondrial transfer. Front. Oncol. 8:344. doi: 10.3389/fonc.2018.00344

Hirao, M., Tamai, N., Tsumaki, N., Yoshikawa, H., and Myoui, A. (2006). Oxygen tension regulates chondrocyte differentiation and function during endochondral ossification. J. Biol. Chem. 281, 31079-31092. doi: 10.1074/jbc.M602296200

Hofmann, A. D., Beyer, M., Krause-Buchholz, U., Wobus, M., Bornhäuser, M., and Rödel, G. (2012). Oxphos supercomplexes as a hallmark of the mitochondrial phenotype of adipogenic differentiated human MSCS. PLOS ONE 7:e35160. doi: 10.1371/journal.pone.0035160

Hole, P. S., Zabkiewicz, J., Munje, C., Newton, Z., Pearn, L., White, P., et al. (2013). Overproduction of NOX-derived ROS in AML promotes proliferation and is associated with defective oxidative stress signaling. Blood 122, 3322-3330. doi: 10.1182/blood-2013-04-491944

Hsu, Y. C., Wu, Y. T., Yu, T. H., and Wei, Y. H. (2016). Mitochondria in mesenchymal stem cell biology and cell therapy: from cellular differentiation to mitochondrial transfer. Semin. Cell Dev. Biol. 52, 119-131. doi: 10.1016/j.semcdb.2016.02.011 
Hu, L., Yin, C., Zhao, F., Ali, A., Ma, J., and Qian, A. (2018). Mesenchymal stem cells: cell fate decision to osteoblast or adipocyte and application in osteoporosis treatment. Int. J. Mol. Sci. 19:360. doi: 10.3390/ijms19020360

Huang, P. L., Chen, Y. C., Chen, L. H., Juan, C. C., Ku, H. H., Wang, S. T., et al. (2011). PGC-1 $\alpha$ mediates differentiation of mesenchymal stem cells to brown adipose cells. J. Atheroscler. Thromb. 18, 966-980. doi: 10.5551/jat.7401

Inoue, T., Hiratsuka, M., Osaki, M., Yamada, H., Kishimoto, I., Yamaguchi, S., et al. (2007). SIRT2, a tubulin deacetylase, acts to block the entry to chromosome condensation in response to mitotic stress. Oncogene 26, 945-957. doi: 10.1038/sj.onc.1209857

Ippolito, L., Morandi, A., Taddei, M. L., Parri, M., Comito, G., Iscaro, A., et al. (2019). Cancer-associated fibroblasts promote prostate cancer malignancy via metabolic rewiring and mitochondrial transfer. Oncogene 38, 5339-5355. doi: 10.1038/s41388-019-0805-7

Ishikawa, E. T., Gonzalez-Nieto, D., Ghiaur, G., Dunn, S. K., Ficker, A. M., Murali, B., et al. (2012). Connexin-43 prevents hematopoietic stem cell senescence through transfer of reactive oxygen species to bone marrow stromal cells. Proc. Natl. Acad. Sci. U.S.A. 109, 9071-9076. doi: 10.1073/pnas.1120358109

Islam, M. N., Das, S. R., Emin, M. T., Wei, M., Sun, L., Westphalen, K., et al. (2012). Mitochondrial transfer from bone-marrow-derived stromal cells to pulmonary alveoli protects against acute lung injury. Nat. Med. 18, 759-765. doi: $10.1038 / \mathrm{nm} .2736$

Jackson, M. V., Morrison, T. J., Doherty, D. F., McAuley, D. F., Matthay, M. A., Kissenpfennig, A., et al. (2016). Mitochondrial transfer via tunneling nanotubes is an important mechanism by which mesenchymal stem cells enhance macrophage phagocytosis in the in vitro and in vivo models of ARDS. Stem Cells 34, 2210-2223. doi: 10.1002/stem.2372

Jeon, Y. J., Kim, J., Cho, J. H., Chung, H. M., and Chae, J Il. (2016). Comparative analysis of human mesenchymal stem cells derived from bone marrow, placenta, and adipose tissue as sources of cell therapy. J. Cell. Biochem. 117, 1112-1125. doi: $10.1002 /$ jcb. 25395

Ji, W. K., Hatch, A. L., Merrill, R. A., Strack, S., and Higgs, H. N. (2015). Actin filaments target the oligomeric maturation of the dynamin GTPase Drp1 to mitochondrial fission sites. eLife 4:e11553. doi: 10.7554/eLife.11553.040

Jiang, D., Gao, F., Zhang, Y., Wong, D. S. H., Li, Q., Tse, H. F., et al. (2016). Mitochondrial transfer of mesenchymal stem cells effectively protects corneal epithelial cells from mitochondrial damage. Cell Death Dis. 7:e2467. doi: 10.1038/cddis.2016.358

Jiang, D., Xiong, G., Feng, H., Zhang, Z., Chen, P., Yan, B., et al. (2019). Donation of mitochondria by iPSC-derived mesenchymal stem cells protects retinal ganglion cells against mitochondrial complex I defect-induced degeneration. Theranostics 9, 2395-2410. doi: 10.7150/thno.29422

Jiang, L., Paone, S., Caruso, S., Atkin-Smith, G. K., Phan, T. K., Hulett, M. D., et al. (2017). Determining the contents and cell origins of apoptotic bodies by flow cytometry. Sci. Rep. 7:14444. doi: 10.1038/s41598-017-14305-z

Jing, E., Gesta, S., and Kahn, C. R. (2007). SIRT2 regulates adipocyte differentiation through FoxO1 acetylation/deacetylation. Cell Metab. 6, 105-114. doi: 10.1016/j.cmet.2007.07.003

Khan, W. S., Adesida, A. B., Tew, S. R., Lowe, E. T., and Hardingham, T. E. (2010). Bone marrow-derived mesenchymal stem cells express the pericyte marker 3G5 in culture and show enhanced chondrogenesis in hypoxic conditions. J. Orthop. Res. 28, 834-840. doi: 10.1002/jor.21043

Kiang, J. G. (2016). Adult mesenchymal stem cells and radiation injury. Health Phys. 111, 198-203. doi: 10.1097/HP.0000000000000459

Kondoh, H., Lleonart, M. E., Bernard, D., and Gil, J. (2007). Protection from oxidative stress by enhanced glycolysis; a possible mechanism of cellular immortalization. Histol. Histopathol. 22, 85-90. doi: 10.14670/HH-22.85

Konopleva, M., Konoplev, S., Hu, W., Zaritskey, A. Y., Afanasiev, B. V., and Andreeff, M. (2002). Stromal cells prevent apoptosis of AML cells by up-regulation of anti-apoptotic proteins. Leukemia 16, 1713-1724. doi: $10.1038 /$ sj.leu.2402608

Kopek, B. G., Shtengel, G., Grimm, J. B., Clayton, D. A., and Hess, H. F. (2013). Correlative photoactivated localization and scanning electron microscopy. PLoS ONE 8:e77209. doi: 10.1371/journal.pone.0077209

Koyanagi, M., Brandes, R. P., Haendeler, J., Zeiher, A. M., and Dimmeler, S. (2005). Cell-to-cell connection of endothelial progenitor cells with cardiac myocytes by nanotubes: a novel mechanism for cell fate changes? Circ. Res. 96, 1039-1041. doi: 10.1161/01.RES.0000168650.23479.0c
Krejcik, J., Casneuf, T., Nijhof, I. S., Verbist, B., Bald, J., Plesner, T., et al. (2016). Daratumumab depletes CD38+ immune regulatory cells, promotes Tcell expansion, and skews T-cell repertoire in multiple myeloma. Blood 128, 384-394. doi: 10.1182/blood-2015-12-687749

Krysko, D. V., Agostinis, P., Krysko, O., Garg, A. D., Bachert, C., Lambrecht, B. N., et al. (2011). Emerging role of damage-associated molecular patterns derived from mitochondria in inflammation. Trends Immunol. 32, 157-164. doi: 10.1016/j.it.2011.01.005

Lagadinou, E. D., Sach, A., Callahan, K., Rossi, R. M., Neering, S. J., Minhajuddin, M., et al. (2013). BCL-2 inhibition targets oxidative phosphorylation and selectively eradicates quiescent human leukemia stem cells. Cell Stem Cell 12, 329-341. doi: 10.1016/j.stem.2012.12.013

Li, C., Cheung, M. K. H., Han, S., Zhang, Z., Chen, L., Chen, J., et al. (2019). Mesenchymal stem cells and their mitochondrial transfer: a double-edged sword. Biosci. Rep. 39:BSR20182417. doi: 10.1042/BSR20182417

Li, C. J., Sun, L. Y., and Pang, C. Y. (2015). Synergistic protection of $\mathrm{N}$-acetylcysteine and ascorbic acid 2-phosphate on human mesenchymal stem cells against mitoptosis, necroptosis and apoptosis. Sci. Rep. 5:9819. doi: 10.1038/srep09819

Li, G., Chan, C., Wang, H., and Kung, H. (2011). Proteomic analysis of human mesenchymal stem cells. Methods Mol. Biol. 698, 443-457. doi: 10.1007/978-1-60761-999-4_32

Li, M., Yan, J., Chen, X., Tam, W., Zhou, L., Liu, T., et al. (2018). Spontaneous upregulation of SIRT1 during osteogenesis contributes to stem cells' resistance to oxidative stress. J. Cell. Biochem. 119, 4928-4944. doi: 10.1002/jcb. 26730

Li, X., Hong, Y., He, H., Jiang, G., You, W., Liang, X., et al. (2019). FGF21 mediates mesenchymal stem cell senescence via regulation of mitochondrial dynamics. Oxid. Med. Cell. Longev. 2019:4915149. doi: 10.1155/2019/4915149

Li, X., Michaeloudes, C., Zhang, Y., Wiegman, C. H., Adcock, I. M., Lian, Q., et al. (2018). Mesenchymal stem cells alleviate oxidative stress-induced mitochondrial dysfunction in the airways. J. Allergy Clin. Immunol. 141, 1634-1645.e5. doi: 10.1016/j.jaci.2017.08.017

Li, X., Zhang, Y., Yeung, S. C., Liang, Y., Liang, X., Ding, Y., et al. (2014). Mitochondrial transfer of induced pluripotent stem cell-derived mesenchymal stem cells to airway epithelial cells attenuates cigarette smoke-induced damage. Am. J. Respir. Cell Mol. Biol. 51, 455-465. doi: 10.1165/rcmb.20130529OC

Lin, C. H., Li, N. T., Cheng, H. S., and Yen, M. L. (2018). Oxidative stress induces imbalance of adipogenic/osteoblastic lineage commitment in mesenchymal stem cells through decreasing SIRT1 functions. J. Cell. Mol. Med. 22, 786-796. doi: $10.1111 / \mathrm{jcmm} .13356$

Lin, H. Y., Liou, C. W., Chen, S. D., Hsu, T. Y., and Chuang, J. H., Wang, P. W., et al. (2015). Mitochondrial transfer from Wharton's jelly-derived mesenchymal stem cells to mitochondria-defective cells recaptures impaired mitochondrial function. Mitochondrion 22, 31-44. doi: 10.1016/j.mito.2015.02.006

Lin, Q., Lee, Y. J., and Yun, Z. (2006). Differentiation arrest by hypoxia. J. Biol. Chem. 281, 30678-30683. doi: 10.1074/jbc.C600120200

Liu, A., and Curran, M. A. (2020). Tumor hypermetabolism confers resistance to immunotherapy. Semin. Cancer Biol. 65, 155-163. doi: 10.1016/j.semcancer.2020.01.009

Liu, K., Ji, K., Guo, L., Wu, W., Lu, H., Shan, P., et al. (2014). Mesenchymal stem cells rescue injured endothelial cells in an in vitro ischemia-reperfusion model via tunneling nanotube like structure-mediated mitochondrial transfer. Microvasc. Res. 92, 10-18. doi: 10.1016/j.mvr.2014.01.008

Liu, T., Han, C., Wang, S., Fang, P., Ma, Z., Xu, L., et al. (2019). Cancer-associated fibroblasts: an emerging target of anti-cancer immunotherapy. J. Hematol. Oncol. 12:86. doi: 10.1186/s13045-019-0770-1

Lombard, D. B., Alt, F. W., Cheng, H.-L., Bunkenborg, J., Streeper, R. S., Mostoslavsky, R., et al. (2007). Mammalian Sir2 homolog SIRT3 regulates global mitochondrial lysine acetylation. Mol. Cell. Biol. 27, 8807-8814. doi: 10.1128/MCB.01636-07

Losón, O. C., Song, Z., Chen, H., and Chan, D. C. (2013). Fis1, Mff, MiD49, and MiD51 mediate Drp1 recruitment in mitochondrial fission. Mol. Biol. Cell 24, 659-667. doi: 10.1091/mbc.e12-10-0721

Lou, E., Fujisawa, S., Morozov, A., Barlas, A., Romin, Y., Dogan, Y., et al. (2012). Tunneling nanotubes provide a unique conduit for intercellular transfer 
of cellular contents in human malignant pleural mesothelioma. PLoS ONE 7:e33093. doi: 10.1371/journal.pone.0033093

Lu, J., Zheng, X., Li, F., Yu, Y., Chen, Z., Liu, Z., et al. (2017). Tunneling nanotubes promote intercellular mitochondria transfer followed by increased invasiveness in bladder cancer cells. Oncotarget 8, 15539-15552. doi: 10.18632/oncotarget.14695

Luengo, A., Gui, D. Y., and Vander Heiden, M. G. (2017). Targeting metabolism for cancer therapy. Cell Chem. Biol. 24, 1161-1180. doi: 10.1016/j.chembiol.2017.08.028

Lv, Y. J., Yang, Y., Sui, B. D., Hu, C. H., Zhao, P., Liao, L., et al. (2018). Resveratrol counteracts bone loss via mitofilin-mediated osteogenic improvement of mesenchymal stem cells in senescence-accelerated mice. Theranostics 8, 2387-2406. doi: 10.7150/thno.23620

Mahrouf-Yorgov, M., Augeul, L., Da Silva, C. C., Jourdan, M., Rigolet, M., Manin, S., et al. (2017). Mesenchymal stem cells sense mitochondria released from damaged cells as danger signals to activate their rescue properties. Cell Death Differ. 24, 1224-1238. doi: 10.1038/cdd.2017.51

Marlein, C. R., Piddock, R. E., Mistry, J. J., Zaitseva, L., Hellmich, C., Horton, R. H., et al. (2019). CD38-driven mitochondrial trafficking promotes bioenergetic plasticity in multiple myeloma. Cancer Res. 79, 2285-2297. doi: 10.1158/0008-5472.CAN-18-0773

Marlein, C. R., Zaitseva, L., Piddock, R. E., Raso-Barnett, L., Scott, M. A., Ingham, C. J., et al. (2018). PGC-1 $\alpha$ driven mitochondrial biogenesis in stromal cells underpins mitochondrial trafficking to leukemic blasts. Leukemia 32, 2073-2077. doi: 10.1038/s41375-018-0221-y

Marlein, C. R., Zaitseva, L., Piddock, R. E., Robinson, S. D., Edwards, D. R., Shafat, M. S., et al. (2017). NADPH oxidase-2 derived superoxide drives mitochondrial transfer from bone marrow stromal cells to leukemic blasts. Blood 130, 1649-1660. doi: 10.1182/blood-2017-03-772939

Martin, T., Strickland, S., Glenn, M., Charpentier, E., Guillemin, H., Hsu, K., et al. (2019). Phase I trial of isatuximab monotherapy in the treatment of refractory multiple myeloma. Blood Cancer J. 9:41. doi: 10.1038/s41408-019-0198-4

Méndez-Ferrer, S., Bonnet, D., Steensma, D. P., Hasserjian, R. P., Ghobrial, I. M., Gribben, J. G., et al. (2020). Bone marrow niches in haematological malignancies. Nat. Rev. Cancer 20, 285-298. doi: 10.1038/s41568-020-0245-2

Minciacchi, V. R., You, S., Spinelli, C., Morley, S., Zandian, M., Aspuria, P. J., et al. (2015). Large oncosomes contain distinct protein cargo and represent a separate functional class of tumor-derived extracellular vesicles. Oncotarget 6, 11327-11341. doi: 10.18632/oncotarget.3598

Mishra, P., and Chan, D. C. (2016). Metabolic regulation of mitochondrial dynamics. J. Cell Biol. 212, 379-387. doi: 10.1083/jcb.201511036

Mistry, J. J., Hellmich, C., Moore, J. A., Marlein, C. R., Pillinger, G., Collins, A., et al. (2019). Daratumumab inhibits AML metabolic capacity and tumor growth through inhibition of CD38 mediated mitochondrial transfer from bone marrow stromal cells to blasts in the leukemic microenvironment. Blood 134, 1385-1385. doi: 10.1182/blood-2019-128592

Mohyeldin, A., Garzón-Muvdi, T., and Quiñones-Hinojosa, A. (2010). Oxygen in stem cell biology: a critical component of the stem cell niche. Cell Stem Cell 7, 150-161. doi: 10.1016/j.stem.2010.07.007

Moreno, L., Perez, C., Zabaleta, A., Manrique, I., Alignani, D., Ajona, D., et al. (2019). The mechanism of action of the anti-CD38 monoclonal antibody isatuximab in multiple myeloma. Clin. Cancer Res. 25, 3176-3187. doi: 10.1158/1078-0432.CCR-18-1597

Morrison, T. J., Jackson, M. V., Cunningham, E. K., Kissenpfennig, A., McAuley, D. F., O'Kane, C. M., et al. (2017). Mesenchymal stromal cells modulate macrophages in clinically relevant lung injury models by extracellular vesicle mitochondrial transfer. Am. J. Respir. Crit. Care Med. 196, 1275-1286. doi: 10.1164/rccm.201701-0170OC

Moschoi, R., Imbert, V., Nebout, M., Chiche, J., Mary, D., Prebet, T., et al. (2016). Protective mitochondrial transfer from bone marrow stromal cells to acute myeloid leukemic cells during chemotherapy. Blood 128, 253-264. doi: 10.1182/blood-2015-07-655860

Nahhas, F., Dryden, S. C., Abrams, J., and Tainsky, M. A. (2007). Mutations in SIRT2 deacetylase which regulate enzymatic activity but not its interaction with HDAC6 and tubulin. Mol. Cell. Biochem. 303, 221-230. doi: 10.1007/s11010-007-9478-6

Nguyen, H., Lee, J. Y., Sanberg, P. R., Napoli, E., and Borlongan, C. V. (2019). Eye opener in stroke: mitochondrial dysfunction and stem cell repair in retinal ischemia. Stroke 50, 2197-2206. doi: 10.1161/STROKEAHA.119. 025249

North, B. J., and Verdin, E. (2004). Sirtuins: Sir2-related NAD-dependent protein deacetylases. Genome Biol. 5:224. doi: 10.1186/gb-2004-5-5-224

Ohkouchi, S., Block, G. J., Katsha, A. M., Kanehira, M., Ebina, M., Kikuchi, T., et al. (2012). Mesenchymal stromal cells protect cancer cells from ROS-induced apoptosis and enhance the warburg effect by secreting STC1. Mol. Ther. 20, 417-423. doi: 10.1038/mt.2011.259

Okada, M., Kim, H. W., Matsu-Ura, K., Wang, Y. G., Xu, M., and Ashraf, M. (2016). Abrogation of Age-induced microRNA-195 rejuvenates the senescent mesenchymal stem cells by reactivating telomerase. Stem Cells 34, 148-159. doi: $10.1002 /$ stem.2211

Önfelt, B., Nedvetzki, S., Benninger, R. K. P., Purbhoo, M. A., and Sowinski, S., Hume, A. N., et al. (2006). Structurally distinct membrane nanotubes between human macrophages support long-distance vesicular traffic or surfing of bacteria. J. Immunol. 177, 8476-8483. doi: 10.4049/jimmunol.177.12.8476

Pacak, C. A., Preble, J. M., Kondo, H., Seibel, P., Levitsky, S., Del Nido, P. J., et al. (2015). Actin-dependent mitochondrial internalization in cardiomyocytes: evidence for rescue of mitochondrial function. Biol. Open 4, 622-626. doi: 10.1242/bio.201511478

Paliwal, S., Chaudhuri, R., Agrawal, A., and Mohanty, S. (2018). Regenerative abilities of mesenchymal stem cells through mitochondrial transfer. J. Biomed. Sci. 25:31. doi: 10.1186/s12929-018-0429-1

Park, J., Chen, Y., Tishkoff, D. X., Peng, C., Tan, M., Dai, L., et al. (2013). SIRT5mediated lysine desuccinylation impacts diverse metabolic pathways. Mol. Cell 50, 919-930. doi: 10.1016/j.molcel.2013.06.001

Pasquier, J., Guerrouahen, B. S., Al Thawadi, H., Ghiabi, P., Maleki, M., AbuKaoud, N., et al. (2013). Preferential transfer of mitochondria from endothelial to cancer cells through tunneling nanotubes modulates chemoresistance. $J$. Transl. Med. 11:94. doi: 10.1186/1479-5876-11-94

Perkins, G., Renken, C., Martone, M. E., Young, S. J., Ellisman, M., and Frey, T. (1997). Electron tomography of neuronal mitochondria: three-dimensional structure and organization of cristae and membrane contacts. J. Struct. Biol. 119, 260-272. doi: 10.1006/jsbi.1997.3885

Pernas, L., and Scorrano, L. (2016). Mito-morphosis: mitochondrial fusion, fission, and cristae remodeling as key mediators of cellular function. Annu. Rev. Physiol. 78, 505-531. doi: 10.1146/annurev-physiol-021115-105011

Phinney, D. G., Di Giuseppe, M., Njah, J., Sala, E., Shiva, S., St Croix, C. M., et al. (2015). Mesenchymal stem cells use extracellular vesicles to outsource mitophagy and shuttle microRNAs. Nat. Commun. 6:8472. doi: $10.1038 /$ ncomms 9472

Pickles, S., Vigié, P., and Youle, R. J. (2018). Mitophagy and quality control mechanisms in mitochondrial maintenance. Curr. Biol. 28, R170-R185. doi: 10.1016/j.cub.2018.01.004

Pietilä, M., Palomäki, S., Lehtonen, S., Ritamo, I., Valmu, L., Nystedt, J., et al. (2012). Mitochondrial function and energy metabolism in umbilical cord blood- and bone marrow-derived mesenchymal stem cells. Stem Cells Dev. 21, 575-588. doi: 10.1089/scd.2011.0023

Pievani, A., Biondi, M., Tomasoni, C., Biondi, A., and Serafini, M. (2020). Location first: targeting acute myeloid leukemia within its niche. J. Clin. Med. 9:1513. doi: $10.3390 /$ jcm 9051513

Pilling, A. D., Horiuchi, D., Lively, C. M., and Saxton, W. M. (2006). Kinesin-1 and dynein are the primary motors for fast transport of mitochondria in drosophila motor axons. Mol. Biol. Cell 17, 2057-2068. doi: 10.1091/mbc.e05-06-0526

Pitt, J. M., Kroemer, G., and Zitvogel, L. (2016). Extracellular vesicles: masters of intercellular communication and potential clinical interventions. J. Clin. Invest. 126, 1139-1143. doi: 10.1172/JCI87316

Plotnikov, E. Y., Khryapenkova, T. G., Galkina, S. I., Sukhikh, G. T., and Zorov, D. B. (2010). Cytoplasm and organelle transfer between mesenchymal multipotent stromal cells and renal tubular cells in co-culture. Exp. Cell Res. 316, 2447-2455. doi: 10.1016/j.yexcr.2010.06.009

Plotnikov, E. Y., Khryapenkova, T. G., Vasileva, A. K., Marey, M. V., Galkina, S. I., Isaev, N. K., et al. (2008). Cell-to-cell cross-talk between mesenchymal stem cells and cardiomyocytes in co-culture. J. Cell. Mol. Med. 12, 1622-1631. doi: 10.1111/j.1582-4934.2007.00205.x

Polak, R., De Rooij, B., Pieters, R., and Den Boer, M. L. (2015). B-cell precursor acute lymphoblastic leukemia cells use tunneling nanotubes to orchestrate their microenvironment. Blood 126, 2404-2414. doi: 10.1182/blood-2015-03-634238 
Quinn, K. P., Sridharan, G. V., Hayden, R. S., Kaplan, D. L., Lee, K., and Georgakoudi, I. (2013). Quantitative metabolic imaging using endogenous fluorescence to detect stem cell differentiation. Sci. Rep. 3:3432. doi: 10.1038/srep03432

Rambold, A. S., Cohen, S., and Lippincott-Schwartz, J. (2015). Fatty acid trafficking in starved cells: regulation by lipid droplet lipolysis, autophagy, and mitochondrial fusion dynamics. Dev. Cell 32, 678-692. doi: 10.1016/j.devcel.2015.01.029

Raposo, G., and Stoorvogel, W. (2013). Extracellular vesicles: exosomes, microvesicles, and friends. J. Cell Biol. 200, 373-383. doi: $10.1083 /$ jcb. 201211138

Rardin, M. J., He, W., Nishida, Y., Newman, J. C., Carrico, C., Danielson, S. R., et al. (2013). SIRT5 regulates the mitochondrial lysine succinylome and metabolic networks. Cell Metab. 18, 920-933. doi: 10.1016/j.cmet.2013.11.013

Resnik, N., Erman, A., Veranič, P., and Kreft, M. E. (2019). Triple labelling of actin filaments, intermediate filaments and microtubules for broad application in cell biology: uncovering the cytoskeletal composition in tunneling nanotubes. Histochem. Cell Biol. 152, 311-317. doi: 10.1007/s00418-019-01806-3

Ribeiro-Rodrigues, T. M., Martins-Marques, T., Morel, S., Kwak, B. R., and Girão, H. (2017). Role of connexin 43 in different forms of intercellular communication-gap junctions, extracellular vesicles and tunnelling nanotubes. J. Cell Sci. 130, 3619-3630. doi: 10.1242/jcs.200667

Rodriguez, A., Nakhle, J., and Griessinger, E. (2018). Intercellular mitochondria trafficking highlighting the dual role of mesenchymal stem cells as both sensors and rescuers of tissue injury. Cell Cycle 17, 712-721. doi: 10.1080/15384101.2018.1445906

Rustom, A., Saffrich, R., Markovic, I., Walther, P., and Gerdes, H. H. (2004). Nanotubular highways for intercellular organelle transport. Science 303, 1007-1010. doi: 10.1126/science.1093133

Saleh, M., Shamsasanjan, K., Movassaghpourakbari, A., Akbarzadehlaleh, P., and Molaeipour, Z. (2015). The impact of mesenchymal stem cells on differentiation of hematopoietic stem cells. Adv. Pharm. Bull. 5, 299-304. doi: 10.15171/apb.2015.042

Samudio, I., Fiegl, M., McQueen, T., Clise-Dwyer, K., and Andreeff, M. (2008). The warburg effect in leukemia-stroma cocultures is mediated by mitochondrial uncoupling associated with uncoupling protein 2 activation. Cancer Res. 68, 5198-5205. doi: 10.1158/0008-5472.CAN-08-0555

Schichor, C., Albrecht, V., Korte, B., Buchner, A., Riesenberg, R., Mysliwietz, J., et al. (2012). Mesenchymal stem cells and glioma cells form a structural as well as a functional syncytium in vitro. Exp. Neurol. 234, 208-219. doi: 10.1016/j.expneurol.2011.12.033

Semenza, G. L. (1998). Hypoxia-inducible factor 1: master regulator of $\mathrm{O} 2$ homeostasis. Curr. Opin. Genet. Dev. 8, 588-594. doi: 10.1016/S0959-437X(98)80016-6

Seo, B. J., Yoon, S. H., and Do, J. T. (2018). Mitochondrial dynamics in stem cells and differentiation. Int. J. Mol. Sci. 19:3893. doi: 10.3390/ijms19123893

Shafat, M. S., Oellerich, T., Mohr, S., Robinson, S. D., Edwards, D. R., Marlein, C. R., et al. (2017). Leukemic blasts program bone marrow adipocytes to generate a protumoral microenvironment. Blood 129, 1320-1332. doi: 10.1182/blood-2016-08-734798

Shilagardi, K., Li, S., Luo, F., Marikar, F., Duan, R., Jin, P., et al. (2013). Actin-propelled invasive membrane protrusions promote fusogenic protein engagement during cell-cell fusion. Science 340, 359-363. doi: $10.1126 /$ science. 1234781

Si, X., Chen, W., Guo, X., Chen, L., Wang, G., Xu, Y., et al. (2013). Activation of GSK3 $\beta$ by Sirt 2 is required for early lineage commitment of mouse embryonic stem cell. PLoS ONE 8, 2-9. doi: 10.1371/journal.pone.0076699

Sillar, J. R., Germon, Z. P., De Iuliis, G. N., and Dun, M. D. (2019). The role of reactive oxygen species in acute myeloid leukaemia. Int. J. Mol. Sci. 20, 1-20. doi: 10.3390/ijms20236003

Simic, P., Zainabadi, K., Bell, E., Sykes, D. B., Saez, B., Lotinun, S., et al. (2013). SIRT1 regulates differentiation of mesenchymal stem cells by deacetylating B-catenin. EMBO Mol. Med. 5, 430-440. doi: 10.1002/emmm.201201606

Sinclair, K. A., Yerkovich, S. T., Hopkins, P. M. A., and Chambers, D. C. (2016). Characterization of intercellular communication and mitochondrial donation by mesenchymal stromal cells derived from the human lung. Stem Cell Res. Ther. 7, 1-10. doi: 10.1186/s13287-016-0354-8
Škrtić, M., Sriskanthadevan, S., Jhas, B., Gebbia, M., Wang, X., Wang, Z., et al. (2011). Inhibition of mitochondrial translation as a therapeutic strategy for human acute myeloid leukemia. Cancer Cell 20, 674-688. doi: 10.1016/j.ccr.2011.10.015

Spees, J. L., Olson, S. D., Whitney, M. J., and Prockop, D. J. (2006). Mitochondrial transfer between cells can rescue aerobic respiration. Proc. Natl. Acad. Sci. U.S.A. 103, 1283-1288. doi: 10.1073/pnas.0510511103

Stab, B. R., Martinez, L., Grismaldo, A., Lerma, A., Gutiérrez, M. L., Barrera, L. A., et al. (2016). Mitochondrial functional changes characterization in young and senescent human adipose derived MSCs. Front. Aging Neurosci. 8, 1-10. doi: 10.3389/fnagi.2016.00299

Suganuma, K., Miwa, H., Imai, N., Shikami, M., Gotou, M., Goto, M., et al. (2010). Energy metabolism of leukemia cells: glycolysis versus oxidative phosphorylation. Leuk. Lymphoma 51, 2112-2119. doi: 10.3109/10428194.2010.512966

Sun, H., Wu, Y., Fu, D., Liu, Y., and Huang, C. (2014). SIRT6 regulates osteogenic differentiation of rat bone marrow mesenchymal stem cells partially via suppressing the nuclear factor-кB signaling pathway. Stem Cells 32, 1943-1955. doi: $10.1002 /$ stem. 1671

Tahara, E. B., Navarete, F. D. T., and Kowaltowski, A. J. (2009). Tissue, substrate-, and site-specific characteristics of mitochondrial reactive oxygen species generation. Free Radic. Biol. Med. 46, 1283-1297. doi: 10.1016/j.freeradbiomed.2009.02.008

Tan, A. S., Baty, J. W., Dong, L. F., Bezawork-Geleta, A., Endaya, B., Goodwin, J., et al. (2015). Mitochondrial genome acquisition restores respiratory function and tumorigenic potential of cancer cells without mitochondrial DNA. Cell Metab. 21, 81-94. doi: 10.1016/j.cmet.2014.12.003

Tormos, K. V., Anso, E., Hamanaka, R. B., Eisenbart, J., Joseph, J., Kalyanaraman, B., et al. (2011). Mitochondrial complex III ROS regulate adipocyte differentiation. Cell Metab. 14, 537-544. doi: 10.1016/j.cmet.2011.08.007

Torralba, D., Baixauli, F., and Sánchez-Madrid, F. (2016). Mitochondria know no boundaries: mechanisms and functions of intercellular mitochondrial transfer. Front. Cell Dev. Biol. 4:107. doi: 10.3389/fcell.2016.00107

Uccelli, A., Moretta, L., and Pistoia, V. (2008). Mesenchymal stem cells in health and disease. Nat. Rev. Immunol. 8, 726-736. doi: 10.1038/nri2395

Ullah, I., Subbarao, R. B., and Rho, G. J. (2015). Human mesenchymal stem cells-current trends and future prospective. Biosci. Rep. 35:e00191. doi: 10.1042/BSR20150025

Vallabhaneni, K. C., Haller, H., and Dumler, I. (2012). Vascular smooth muscle cells initiate proliferation of mesenchymal stem cells by mitochondrial transfer via tunneling nanotubes. Stem Cells Dev. 21, 3104-3113. doi: $10.1089 / \mathrm{scd} .2011 .0691$

Van Niel, G., D'Angelo, G., and Raposo, G. (2018). Shedding light on the cell biology of extracellular vesicles. Nat. Rev. Mol. Cell Biol. 19, 213-228. doi: $10.1038 / \mathrm{nrm} .2017 .125$

Ventura-Clapier, R., Garnier, A., and Veksler, V. (2008). Transcriptional control of mitochondrial biogenesis: the central role of PGC-1 $\alpha$. Cardiovasc. Res. 79, 208-217. doi: 10.1093/cvr/cvn098

Vogel, F., Bornhövd, C., Neupert, W., and Reichert, A. S. (2006). Dynamic subcompartmentalization of the mitochondrial inner membrane. J. Cell Biol. 175, 237-247. doi: 10.1083/jcb.200605138

Vyas, S., Zaganjor, E., and Haigis, M. C. (2016). Mitochondria and cancer. Cell 166, 555-566. doi: 10.1016/j.cell.2016.07.002

Wagegg, M., Gaber, T., Lohanatha, F. L., Hahne, M., Strehl, C., Fangradt, M., et al. (2012). Hypoxia promotes osteogenesis but suppresses adipogenesis of human mesenchymal stromal cells in a hypoxia-inducible factor-1 dependent manner. PLOS ONE 7:e46483. doi: 10.1371/journal.pone.0046483

Wang, F., and Tong, Q. (2009). SIRT2 suppresses adipocyte differentiation by deacetylating FOXO1 and enhancing FOXO1's repressive interaction with PPAR $\gamma$. Mol. Biol. Cell 20, 801-808. doi: 10.1091/mbc.e08-06-0647

Wang, J., Liu, X., Qiu, Y., Shi, Y., Cai, J., Wang, B., et al. (2018). Cell adhesionmediated mitochondria transfer contributes to mesenchymal stem cell-induced chemoresistance on $\mathrm{T}$ cell acute lymphoblastic leukemia cells. J. Hematol. Oncol. 11, 1-13. doi: 10.1186/s13045-018-0554-Z

Wang, Y., Cui, J., Sun, X., and Zhang, Y. (2011). Tunneling-nanotube development in astrocytes depends on p53 activation. Cell Death Differ. 18, 732-742. doi: $10.1038 /$ cdd.2010.147 
Weiss, A. R. R., and Dahlke, M. H. (2019). Immunomodulation by mesenchymal stem cells (MSCs): mechanisms of action of living, apoptotic, and dead MSCs. Front. Immunol. 10:1191. doi: 10.3389/fimmu.2019.01191

Xiao, Y., and Chen, J. (2012). Proteomics approaches in the identification of molecular signatures of mesenchymal stem cells. Adv. Biochem. Eng. Biotechnol. 129, 153-176. doi: 10.1007/10_2012_143

Yang, W., Guo, X., Thein, S., Xu, F., Sugii, S., Baas, P. W., et al. (2013). Regulation of adipogenesis by cytoskeleton remodelling is facilitated by acetyltransferase MEC-17-dependent acetylation of $\alpha$-tubulin. Biochem. J. 449, 606-612. doi: 10.1042/BJ20121121

Yao, Y., Fan, X. L., Jiang, D., Zhang, Y., Li, X., Xu, Z., et al. (2018). Connexin 43-mediated mitochondrial transfer of iPSC-MSCs alleviates asthma inflammation. Stem Cell Rep. 11, 1120-1135. doi: 10.1016/j.stemcr.2018.09.012

Yao, Y., Zeng, Q. X., Deng, X. Q., Tang, G. N., Guo, J. B., Sun, Y. Q., et al. (2015). Connexin 43 upregulation in mouse lungs during ovalbumin-induced asthma. PLoS ONE 10:e0144106. doi: 10.1371/journal.pone.0144106

Yun, Z., Maecker, H. L., Johnson, R. S., and Giaccia, A. J. (2002). Inhibition of PPAR $\gamma 2$ gene expression by the HIF-1-regulated gene DEC1/Stra13: a mechanism for regulation of adipogenesis by hypoxia. Dev. Cell 2, 331-341. doi: 10.1016/S1534-5807(02)00131-4

Zhang, H., Menzies, K. J., and Auwerx, J. (2018). The role of mitochondria in stem cell fate and aging. Development 145:dev143420. doi: 10.1242/dev. 143420

Zhang, P., Liu, Y., Wang, Y., Zhang, M., Lv, L., Zhang, X., et al. (2017). SIRT6 promotes osteogenic differentiation of mesenchymal stem cells through BMP signaling. Sci. Rep. 7, 1-10. doi: 10.1038/s41598-017-10323-z
Zhang, Y., Marsboom, G., Toth, P. T., and Rehman, J. (2013). Mitochondrial respiration regulates adipogenic differentiation of human mesenchymal stem cells. PLOS ONE 8:e77077. doi: 10.1371/journal.pone. 0077077

Zhang, Y., Yu, Z., Jiang, D., Liang, X., Liao, S., Zhang, Z., et al. (2016). iPSC-MSCs with high intrinsic MIRO1 and sensitivity to TNF- $\alpha$ yield efficacious mitochondrial transfer to rescue anthracycline-induced cardiomyopathy. Stem Cell Rep. 7, 749-763. doi: 10.1016/j.stemcr.2016. 08.009

Zhou, B., and Tian, R. (2018). Mitochondrial dysfunction in pathophysiology of heart failure. J. Clin. Invest. 128, 3716-3726. doi: 10.1172/JCI120849

Zick, M., Rabl, R., and Reichert, A. S. (2009). Cristae formation-linking ultrastructure and function of mitochondria. Biochim. Biophys. Acta Mol. Cell Res. 1793, 5-19. doi: 10.1016/j.bbamcr.2008.06.013

Conflict of Interest: The authors declare that the research was conducted in the absence of any commercial or financial relationships that could be construed as a potential conflict of interest.

Copyright (C) 2020 Mohammadalipour, Dumbali and Wenzel. This is an open-access article distributed under the terms of the Creative Commons Attribution License (CC $B Y)$. The use, distribution or reproduction in other forums is permitted, provided the original author(s) and the copyright owner(s) are credited and that the original publication in this journal is cited, in accordance with accepted academic practice. No use, distribution or reproduction is permitted which does not comply with these terms. 